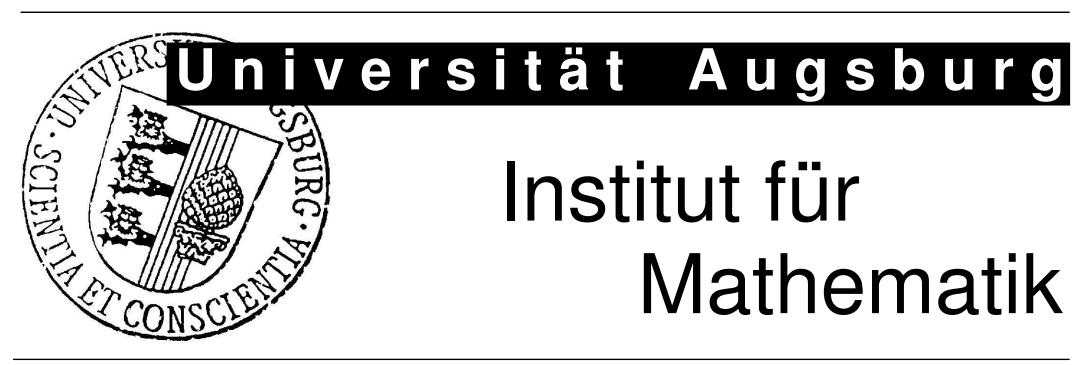

Xuejun Xu, Huangxin Chen, Ronald H.W. Hoppe

Optimality of Local Multilevel Methods on Adaptively Refined Meshes for Elliptic Boundary Value Problems 


\section{Impressum:}

\section{Herausgeber:}

Institut für Mathematik

Universität Augsburg

86135 Augsburg

http://www.math.uni-augsburg.de/pages/de/forschung/preprints.shtml

\section{ViSdP:}

Ronald H.W. Hoppe

Institut für Mathematik

Universität Augsburg

86135 Augsburg

Preprint: Sämtliche Rechte verbleiben den Autoren (C) 2009 


\title{
OPTIMALITY OF LOCAL MULTILEVEL METHODS ON ADAPTIVELY REFINED MESHES FOR ELLIPTIC BOUNDARY VALUE PROBLEMS
}

\author{
XUEJUN XU* , HUANGXIN CHEN*, AND R.H.W. HOPPE ${ }^{\dagger}$
}

\begin{abstract}
A local multilevel product algorithm and its additive version are analyzed for linear systems arising from the application of adaptive finite element methods to second order elliptic boundary value problems. The abstract Schwarz theory is applied to verify uniform convergence of local multilevel methods featuring Jacobi and Gauss-Seidel smoothing only on local nodes. By this abstract theory, convergence estimates can be further derived for the hierarchical basis multigrid method and the hierarchical basis preconditioning method on locally refined meshes, where local smoothing is performed only on new nodes. Numerical experiments confirm the optimality of the suggested algorithms.
\end{abstract}

1. Introduction. Multigrid or multilevel methods belong to the most efficient methods to solve large linear systems arising from the discretization of elliptic boundary value problems by finite element methods. The convergence properties of multigrid methods for conforming finite elements have been studied by many authors (cf., e.g., [9], [10], [11], [8], [14], [19], [24], [32]). The hierarchical basis multilevel method ([34], [35]) and the hierarchical basis multigrid method [6] have been developed by $\mathrm{H}$. Yserentant et al. for finite element methods on quasi-uniform meshes. In particular, using the notions of space decomposition and subspace correction, a unified theory has been established in [32] for a general class of iterative algorithms such as multigrid methods, overlapping domain decomposition methods, and hierarchical basis methods.

In this paper, we study local multilevel methods for adaptive finite element methods (AFEM) applied to second order elliptic boundary value problems. Mesh adaptivity based on a posteriori error estimators has become a powerful tool for solving partial differential equations. It is known that the convergence property of AFEM with the newest vertex bisection algorithm is optimal in the sense that the finite element discretization error is proportional to $N^{-1 / 2}$ in the energy norm, where $N$ is the number of degrees of freedom on the underlying mesh (cf., e.g., [7], [16], [22], [28]). Since the number of nodes per level may not grow exponentially with the mesh levels, as has been pointed out in [23], the number of operations used for multigrid methods with smoothers performed on all nodes can be as large as $O\left(N^{2}\right)$. Therefore, it is interesting to study efficient iterative algorithms to solve the linear systems arising from AFEM procedures. Numerical experiments in [23] indicate the optimality of local multigrid methods performing smoothing only on newly created nodes and their neighbors.

In recent years, some techniques have been developed to handle problems on locally refined meshes. One approach in [20], [21] and [31] is the fast adaptive composite grid (FAC) method, using global and local uniform grids both to define the composite grid problem and to interact for fast solution, which is very suitable for parallel computation. Other approaches have been developed as well such as multilevel adaptive techniques (MLAT) studied in [3], [12], [13], [24], and multigrid methods for locally refined finite element meshes [1], [2], [18], [25], [26]. We emphasize that these locally refined meshes obey restrictive conditions which are not satisfied by the newest vertex bisection algorithm which will be used for adaptivity in this work. As far as AFEM procedures featuring the newest vertex bisection algorithm are concerned, $\mathrm{Wu}$ and Chen [30] have been the first to show that the multigrid V-cycle algorithm performng Gauss-Seidel smoothing on new nodes and those old nodes where the support of the associated nodal basis function has changed can guarantee uniform convergence of the algorithm.

The objective of this paper is to utilize the well-known Schwarz theory [29] to study local multilevel methods with local Jacobi or local Gauss-Seidel smoothing. Within this framework

*LSEC, Institute of Computational Mathematics, Chinese Academy of Sciences, P.O.Box 2719, Beijing, 100190, People's Republic of China(xxj@lsec.cc.ac.cn, chx@lsec.cc.ac.cn). The work of these two authors was supported in parts by the National Basic Research Program of China (Grant No. 2005CB321701) and the National Natural Science Foundation of China (Grant No. 10731060).

${ }^{\dagger}$ Institut für Mathematik, Universität Augsburg, D-86159, Augsburg, Germany (hoppe@math.uni-augsburg.de), and Department of Mathematics, University of Houston, Houston, TX 77204-3008, USA (rohop@math.uh.edu). The work of this author was supported in parts by NSF grants DMS-0707602, DMS-0810156, DMS-0811153, and DMS-0914788. 
we can also derive convergence estimates for the hierarchical basis multigrid method and the hierarchical basis preconditioning method on locally refined meshes, where the local smoothers are performed only on new nodes. In this paper, the main difficulty is how to obtain a global strengthened Cauchy-Schwarz inequality which is a key assumption in the Schwarz theory. We will prove that the global strengthened Cauchy-Schwarz inequality holds true not only for the local Gauss-Seidel iteration, but also for the local Jacobi iteration. Moreover, we point out that the Xu and Zikatanov identity [33], on which the proof in [30] depends, can not be directly used in this paper. The convergence estimate in [30] can only be deduced for multiplicative smoothers and thus is not available for additive smoothers such as the Jacobi iteration. Finally, for the hierarchical basis multigrid method, a nontrivial stability splitting property on locally refined meshes is obtained.

The remainder of this paper is organized as follows: In section 2, we introduce basic notations and briefly review the conforming P1 finite element method on locally refined meshes. In section 3 , we propose a local multilevel product algorithm (or a local multigrid method) and its additive version. In section 4 , we present the abstract theory based on three assumptions whose verification is carried out for local Jacobi and local Gauss-Seidel smoothers, respectively. We further derive and analyze the hierarchical basis multigrid method and the hierarchical basis preconditioning method on locally refined meshes in section 5. Finally, in the last section we present numerical results for some representative test examples that confirm our theoretical analysis.

2. Notations and Preliminaries. Throughout this paper, we adopt standard notations from Lebesgue and Sobolev space theory (cf. e.g. [17]). In particular, we refer to $(\cdot, \cdot)$ as the inner product and to $\|\cdot\|_{1, \Omega}$ as the norm on the Sobolev space $H^{1}(\Omega)$. We further use $A \lesssim B$, if $A \leq C B$ with a positive constant $C$ depending only on the shape regularity of the meshes. $A \approx B$ stands for $A \lesssim B \lesssim A$. For simplicity, we restrict ourselves to the $2 \mathrm{D}$ case.

Given a bounded, polygonal domain $\Omega \subset R^{2}$, we consider the following second order elliptic boundary value problem

$$
\begin{aligned}
\mathcal{L} u:=-\operatorname{div}(a(x) \nabla u) & =f & & \text { in } \Omega, \\
u & =0 & & \text { on } \partial \Omega .
\end{aligned}
$$

The choice of a homogeneous Dirichlet boundary condition is made for ease of presentation only. Similar results are valid for other types of boundary conditions and equation (2.1) with a lower order term as well. We further assume that the coefficient function $a$ and the right-hand side $f$ in (2.1) satisfy the following properties:

(a) $a$ is a measurable function and there exist constants $\beta_{1} \geq \beta_{0}>0$ such that

$$
\beta_{0} \leq a(x) \leq \beta_{1} \quad \text { for almost all } x \in \Omega
$$

(b) $f \in L^{2}(\Omega)$.

The weak formulation of $(2.1)$ and $(2.2)$ is to find $u \in V:=H_{0}^{1}(\Omega)$ such that

$$
a(u, v)=(f, v) \quad, \quad v \in V,
$$

where the bilinear form $a: V \times V \rightarrow \mathbb{R}$ is given by

$$
a(u, v)=(a \nabla u, \nabla v) \quad, \quad u, v \in V .
$$

Since the bilinear form (2.5) is bounded and $V$-elliptic, the existence and uniqueness of the solution of (2.4) follows from the Lax-Milgram theorem.

Throughout this paper, we work with families of shape regular meshes $\left\{\mathcal{T}_{i}, i=0,1, \ldots, J\right\}$, where $\mathcal{T}_{0}$ is an intentionally chosen coarse initial triangulation, the others are obtained by the adaptive procedures using the newest vertex bisection algorithm. It has been proved in [4] that there exists a constant $\theta>0$ such that

$$
\theta_{T} \geq \theta \quad, \quad T \in \mathcal{T}_{i}, i=1,2, \ldots,
$$


where $\theta_{T}$ is the minimum angle of the element $T$. The set of edges on $\mathcal{T}_{i}$ is denoted by $\mathcal{E}_{i}$, and the set of interior and boundary edges by $\mathcal{E}_{i}^{0}$ and $\mathcal{E}_{i}^{\partial \Omega}$, respectively. We refer to $\mathcal{N}_{i}$ as the set of interior nodes of $\mathcal{T}_{i}$. The domain $\Omega_{i}^{z}$ is the union of elements containing $z \in \mathcal{N}_{i}$ and $h_{i}^{z}$ refers to the shortest edge of $\mathcal{E}_{i}\left(\Omega_{i}^{z}\right)$. For any $T \in \mathcal{T}_{i}, h_{i, T}$ stands for the diameter of $T$.

We refer to $V_{J}$ as the conforming $P_{1}$ finite element space

$$
V_{J}=\left\{v_{J} \in V\left|v_{J}\right|_{T} \in P_{1}(T), T \in \mathcal{T}_{J}\right\}
$$

The conforming finite element approximation of (2.4) is to find $u_{J} \in V_{J}$ such that

$$
a\left(u_{J}, v_{J}\right)=\left(f, v_{J}\right) \quad, \quad v_{J} \in V_{J} .
$$

The existence and uniqueness of the solution $u_{J}$ follows again from the Lax-Milgram theorem.

The computation of the solution $u_{J}$ of (2.7) always requires a constructive approach involving the conversion of the variational equation into a matrix equation using a particular basis for $V_{J}$. Suppose that $\left\{\phi_{i}, i=1, \ldots, N\right\}$ is a given basis for $V_{J}$, where $N$ is the dimension of $V_{J}$, and define the matrix $A$ and the vector $F$ via

$$
A_{i j}:=a\left(\phi_{i}, \phi_{j}\right) \quad \text { and } \quad F_{i}:=\left(f, \phi_{i}\right) \quad, \quad i, j=1, \ldots, N .
$$

Then equation (2.7) is equivalent to

$$
A X=F,
$$

where $u_{J}=\sum_{i=1}^{N} u_{i} \phi_{i}$ and $X=\left(u_{i}\right)$.

Bank and Scott [5] have shown that the $\ell_{2}$-condition number of the linear system (2.8) does not necessarily degrade as the mesh is refined locally and can be bounded by

$$
\mathcal{K}_{2}(A) \lesssim N\left(1+\left|\log \left(N h_{\min }^{2}\left(\mathcal{T}_{J}\right)\right)\right|\right),
$$

where $h_{\min }\left(\mathcal{T}_{J}\right)=\min \left\{h_{J, T}: T \in \mathcal{T}_{J}\right\}$. Moreover, the upper bound is sharp.

Based on the estimate (2.9), we know that standard iterative methods for solving the large linear system (2.8) will converge very slowly. The objective of this paper is to design efficient multilevel solvers of optimal computational complexity.

3. Local multilevel methods. In this section, we develop local multilevel methods for solving linear systems arising from AFEM procedures. For any $0 \leq i \leq J$, define $A_{i}: V_{i} \rightarrow V_{i}$, the discretization operator on level $i$, by

$$
\left(A_{i} v, w\right)=a(v, w) \quad, \quad v, w \in V_{i} .
$$

Then the finite element discretization of (2.4) is to find $u_{i} \in V_{i}$ such that

$$
A_{i} u_{i}=f_{i}
$$

where $f_{i} \in V_{i}$ satisfies $\left(f_{i}, v\right)=(f, v), v \in V_{i}$. We also define projections $P_{i}, Q_{i}: V_{J} \rightarrow V_{i}$,

$$
a\left(P_{i} v, w\right)=a(v, w) \quad, \quad\left(Q_{i} v, w\right)=(v, w) \quad, \quad v \in V_{J}, w \in V_{i} .
$$

For any node $z \in \mathcal{N}_{i}$, we use the notation $\varphi_{i}^{z}$ to represent the associated nodal conforming finite element basis function of $V_{i}$. Let $\tilde{\mathcal{N}}_{i}$ be the set of new nodes and those old nodes where the support of the associated basis function has changed (see Figure 3.1), i.e.,

$$
\tilde{\mathcal{N}}_{i}=\left\{z \in \mathcal{N}_{i}: z \in \mathcal{N}_{i} \backslash \mathcal{N}_{i-1} \text { or } z \in \mathcal{N}_{i-1} \text { but } \varphi_{i}^{z} \neq \varphi_{i-1}^{z}\right\}
$$

For convenience, we set $\tilde{\mathcal{N}}_{i}=\left\{x_{i}^{k}, k=1, \ldots, \tilde{n}_{i}\right\}$, where $\tilde{n}_{i}$ is the cardinality of $\tilde{\mathcal{N}}_{i}$, and we denote by $\phi_{i}^{k}=\phi_{i}^{x_{i}^{k}}$ the conforming $P_{1}$ finite element basis function associated with $x_{i}^{k}$. We define local projections $P_{i}^{k}, Q_{i}^{k}: V_{J} \rightarrow V_{i}^{k}:=\operatorname{span}\left\{\phi_{i}^{k}\right\}$ by

$$
a\left(P_{i}^{k} v, \phi_{i}^{k}\right)=a\left(v, \phi_{i}^{k}\right) \quad, \quad\left(Q_{i}^{k} v, \phi_{i}^{k}\right)=\left(v, \phi_{i}^{k}\right) \quad, \quad v \in V_{J},
$$




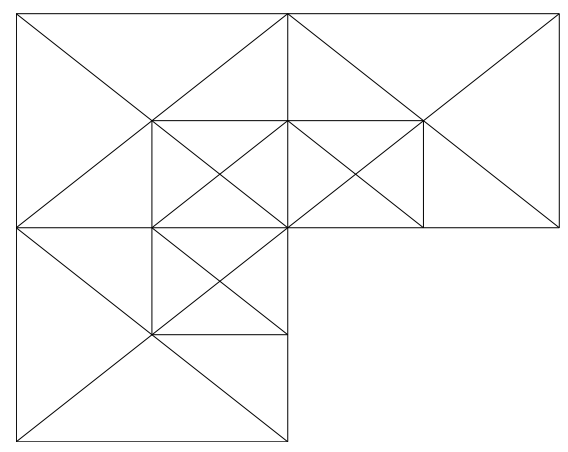

The coarse level $\mathcal{T}_{i-1}$

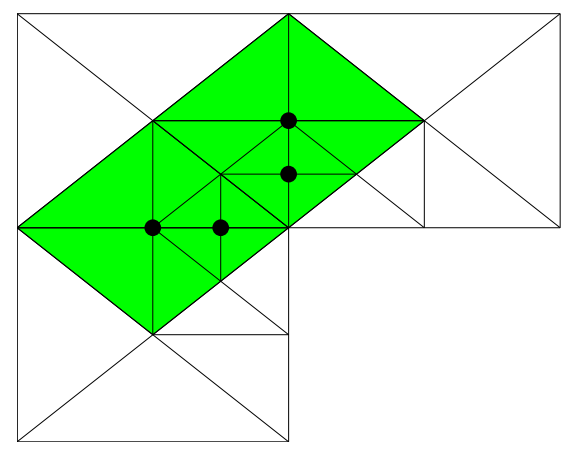

The fine level $\mathcal{T}_{i}$

FIG 3.1. Illustration of $\tilde{\mathcal{N}}_{i}$ : the big dots in the right figure refer to $\tilde{\mathcal{N}}_{i}$.

and $A_{i}^{k}: V_{i}^{k} \rightarrow V_{i}^{k}$ by

$$
\left(A_{i}^{k} v, \phi_{i}^{k}\right)=a\left(v, \phi_{i}^{k}\right) \quad, \quad v \in V_{i}^{k} .
$$

We further refer to $R_{i}: V_{i} \rightarrow V_{i}$ as a local smoothing operator which is assumed to be nonnegative, symmetric or nonsymmetric with respect to the inner product $(\cdot, \cdot)$. For $i=1, \ldots, J, R_{i}$ is only performed on local nodes $\tilde{\mathcal{N}}_{i}$. The linear system on the coarsest mesh is solved directly, i.e., $R_{0}=A_{0}^{-1}$.

We now state the local multilevel algorithms for AFEM as follows.

\section{ALGORITHM 3.1. Local Multigrid algorithm(LMG)}

The standard Multigrid V-cycle algorithm solves (3.1) by the following iterative method:

$$
u_{i}^{n+1}=u_{i}^{n}+B_{i}\left(f_{i}-A_{i} u_{i}^{n}\right) .
$$

The operators $B_{i}: V_{i} \rightarrow V_{i}, 0 \leq i \leq J$ are recursively defined as follows:

(V-cycle algorithm). Let $B_{0}=A_{0}^{-1}$. For $i \geq 1$ and $g \in V_{i}$, we define $B_{i} g=x_{3}$.

(i). Pre-smoothing: $x_{1}=R_{i}^{t} f$,

(ii). Correction: $x_{2}=x_{1}+B_{i-1} Q_{i-1}\left(g-A_{i} x_{1}\right)$,

(iii). Post-smoothing: $x_{3}=x_{2}+R_{i}\left(g-A_{i} x_{2}\right)$.

\section{Algorithm 3.2. Local multilevel additive algorithm(LMAA)}

For $B_{J}=\sum_{i=0}^{J} R_{i} Q_{i}$, find $u_{J} \in V_{J}$ such that

$$
B_{J} A_{J} u_{J}=B_{J} f_{J} .
$$

REMARK 3.1. The $C G$ method can be used to solve the new problem (3.2), if $B_{J} A_{J}$ is symmetric with respect to the inner product $a(\cdot, \cdot)$.

4. The abstract theory. In this section, we present the abstract theory concerned with the convergence of local multilevel methods for linear systems arising from AFEM procedures. We will use the well-known Schwarz theory developed in [29], [32] and [36] to analyze the algorithms LMG and LMAA. To this end, we set

$$
T_{i}:=R_{i} A_{i} P_{i}, i=0,1, \ldots, J, \quad \text { and } \quad T:=\sum_{i=0}^{J} T_{i} .
$$

The abstract theory provides an estimate for the norm of the error operator

$$
E=\left(I-T_{J}\right) \cdots\left(I-T_{1}\right)\left(I-T_{0}\right)=\prod_{i=0}^{J}\left(I-T_{i}\right),
$$


where $I$ is the identity operator in $V_{J}$. Convergence estimates for algorithm 3.1 are obtained by upper bounds for $E$ in the energy norm $\|\cdot\|_{a}:=a(\cdot, \cdot)^{\frac{1}{2}}$. To this end, we impose the following assumptions:

(A1). Each operator $T_{i}$ is nonnegative with respect to the inner product $a(\cdot, \cdot)$, and there exists a positive constant $\omega_{i}<2$ such that

$$
a\left(T_{i} v, T_{i} v\right) \leq \omega_{i} a\left(T_{i} v, v\right) \quad, \quad v \in V_{J}, i=0,1, \ldots, J
$$

(A2). Stability: There exists a constant $K_{0}$ such that

$$
a(v, v) \leq K_{0} a(T v, v) \quad, \quad v \in V_{J}
$$

(A3). Global strengthened Cauchy-Schwarz inequality: There exists a constant $K_{1}$ such that

$$
\sum_{i=0}^{J} \sum_{j=0}^{i-1} a\left(T_{i} v, T_{j} u\right) \leq K_{1}\left(\sum_{i=0}^{J} a\left(T_{i} v, v\right)\right)^{1 / 2}\left(\sum_{j=0}^{J} a\left(T_{j} u, u\right)\right)^{1 / 2} \quad, \quad v, u \in V_{J} .
$$

We remark that the following inequality should also be verified for local Jacobi and local GaussSeidel smoothers, which can not be deduced by the Cauchy-Schwarz inequality for $T_{i}$ directly.

$$
\sum_{i=0}^{J} a\left(T_{i} v, u\right) \leq K_{2}\left(\sum_{i=0}^{J} a\left(T_{i} v, v\right)\right)^{1 / 2}\left(\sum_{i=0}^{J} a\left(T_{i} u, u\right)\right)^{1 / 2} \quad, \quad v, u \in V_{J} .
$$

THEOREM 4.1. If assumptions A1-A3 are satisfied, then the norm of the error operator $E$ can be bounded as follows (cf. [29], [32], [36]):

$$
a(E v, E v) \leq \delta a(v, v) \quad, \quad v \in V_{J},
$$

where $\delta=1-(2-\omega) /\left(K_{0}\left(K_{1}+K_{2}\right)^{2}\right), \omega=\max _{i}\left\{\omega_{i}\right\}$. Hence, for algorithm 3.1 there holds

$$
\left\|I-B_{J} A_{J}\right\|_{a}=\left\|E E^{*}\right\|_{a} \leq \delta .
$$

For the additive multilevel algorithm 3.2, the following theorem provides a spectral estimate for the operator $T=\sum_{i=0}^{J} T_{i}$ when $T$ is symmetric with respect to $a(\cdot, \cdot)$.

TheOREM 4.2. If $T$ is symmetric with respect to $a(\cdot, \cdot)$ and assumptions A1-A3 hold true, then we have (cf. [29], [32], [36])

$$
\frac{1}{K_{0}} a(v, v) \leq a(T v, v) \leq\left(2 K_{1}+\omega\right) a(v, v) \quad, \quad v \in V_{J}
$$

We begin to apply the abstract theory to algorithm 3.1 and algorithm 3.2 by verifying assumptions A1-A3 for adaptive finite element methods. There are two classes of smoothers for $R_{i}$, Jacobi and Gauss-Seidel iterations, which will be investigated separately.

4.1. Local Jacobi smoother. First, we consider the decomposition of $v \in V_{J}$ according to

$$
v=\sum_{i=0}^{J} v_{i} \quad, \quad v_{0}=\Pi_{0} v \quad, \quad v_{i}=\left(\Pi_{i}-\Pi_{i-1}\right) v \quad, \quad i=1, \ldots, J
$$

where $\Pi_{i}: V_{J} \rightarrow V_{i}$ is the Scott-Zhang interpolation operator [27].

The local Jacobi smoother is defined as an additive smoother (cf. [9]):

$$
R_{i}:=\gamma \sum_{k=1}^{\tilde{n}_{i}}\left(A_{i}^{k}\right)^{-1} Q_{i}^{k}
$$


where $\gamma$ is an appropriately chosen positive scaling factor. Due to the definition of $R_{i}$, we have

$$
T_{0}=P_{0} \quad, \quad T_{i}=R_{i} A_{i} P_{i}=\gamma \sum_{k=1}^{\tilde{n}_{i}} P_{i}^{k} \quad, \quad i=1, \ldots, J .
$$

It is easy to deduce that $K_{2}=1$ in (4.1) in the Jacobi case. Therefore, we only need to verify assumptions A1-A3. Actually,

$$
\begin{aligned}
a(T v, u) & =a\left(P_{0} v, P_{0} u\right)+\gamma \sum_{i=1}^{J} \sum_{k=1}^{\tilde{n}_{i}} a\left(P_{i}^{k} v, P_{i}^{k} u\right) \\
& \leq a\left(P_{0} v, v\right)^{1 / 2} a\left(P_{0} u, u\right)^{1 / 2}+\gamma \sum_{i=1}^{J} \sum_{k=1}^{\tilde{n}_{i}} a\left(P_{i}^{k} v, v\right)^{1 / 2} a\left(P_{i}^{k} u, u\right)^{1 / 2} \\
& \leq\left(a\left(P_{0} v, v\right)+\gamma \sum_{i=1}^{J} \sum_{k=1}^{\tilde{n}_{i}} a\left(P_{i}^{k} v, v\right)\right)^{1 / 2}\left(a\left(P_{0} u, u\right)+\gamma \sum_{i=1}^{J} \sum_{k=1}^{\tilde{n}_{i}} a\left(P_{i}^{k} u, u\right)\right)^{1 / 2} \\
& =\left(\sum_{i=0}^{J} a\left(T_{i} v, v\right)\right)^{1 / 2}\left(\sum_{i=0}^{J} a\left(T_{i} u, u\right)\right)^{1 / 2} .
\end{aligned}
$$

4.1.1. Verification of assumption A1. Assumption A1 is easily obtained for $T_{0}$. We analyze the case $i \geq 1$.

Lemma 4.1. Let $T_{i}, i \geq 1$, be defined by (4.4). Then, there holds

$$
a\left(T_{i} v, T_{i} v\right) \leq \omega_{i} a\left(T_{i} v, v\right) \quad, \quad v \in V_{J} \quad, \quad \omega_{i}<2 .
$$

Moreover, $T_{i}$ is symmetric and nonnegative on $V_{J}$. Therefore, assumption $\mathbf{A} 1$ is satisfied.

Proof. Following (4.4), for $v, w \in V_{J}$ we deduce

$$
a\left(T_{i} v, w\right)=a\left(R_{i} A_{i} P_{i} v, w\right)=a\left(R_{i} A_{i} P_{i} v, P_{i} w\right)=\left(R_{i} A_{i} P_{i} v, A_{i} P_{i} w\right) .
$$

In view of the definition of $R_{i}$ by (4.3), it follows that $R_{i}$ is symmetric and nonnegative in $V_{i}$. Hence, $T_{i}$ is symmetric and nonnegative in $V_{J}$. We set

$$
K_{i}^{k}=\left\{P_{i}^{m}: \operatorname{supp}\left(P_{i}^{k} v\right) \cap \operatorname{supp}\left(P_{i}^{m} v\right) \neq \varnothing, v \in V_{i}, m=1, \ldots, \tilde{n}_{i}\right\} .
$$

Then, the cardinality of $K_{i}^{k}$ is bounded by a constant depending only on the minimum angle $\theta$ in (2.6). Based on this fact and Hölder's inequality, there exists a constant $C_{i}$ such that

$$
\sum_{k, m=1}^{\tilde{n}_{i}}\left|a\left(P_{i}^{k} v, P_{i}^{m} v\right)\right| \leq C_{i} \sum_{k=1}^{\tilde{n}_{i}} a\left(P_{i}^{k} v, P_{i}^{k} v\right) \quad, \quad v \in V_{i} .
$$

By the definition of $T_{i}$ in (4.4) and observing (4.6), for $v \in V_{J}$ we obtain

$$
\begin{aligned}
a\left(T_{i} v, T_{i} v\right) & =\gamma^{2} a\left(\sum_{k=1}^{\tilde{n}_{i}} P_{i}^{k} v, \sum_{k=1}^{\tilde{n}_{i}} P_{i}^{k} v\right) \leq \gamma^{2} \sum_{k, m=1}^{\tilde{n}_{i}}\left|a\left(P_{i}^{k} v, P_{i}^{m} v\right)\right| \\
& \leq \gamma^{2} C_{i} \sum_{k=1}^{\tilde{n}_{i}} a\left(P_{i}^{k} v, P_{i}^{k} v\right)=\gamma^{2} C_{i} \sum_{k=1}^{\tilde{n}_{i}} a\left(P_{i}^{k} v, v\right)=\gamma C_{i} a\left(T_{i} v, v\right) .
\end{aligned}
$$

The proof is completed by setting $\omega_{i}=\gamma C_{i}$ and choosing $0<\gamma<1$ such that $\omega_{i}<2$. 
4.1.2. Verification of assumption A2. We will rely on the decomposition (4.2).

Lemma 4.2. Let $\left\{T_{i}, i=0,1, \ldots, J\right\}$ be defined by (4.4). Then, there exists a constant $K_{0}$ such that

$$
a(v, v) \leq K_{0} a(T v, v) \quad, \quad v \in V_{J} .
$$

Proof. Due to (4.2), we have

$$
a(v, v)=\sum_{i=0}^{J} a\left(v_{i}, v\right)
$$

and for $i=1, \ldots, J$, we obtain

$$
\begin{aligned}
a\left(v_{i}, v\right) & =\sum_{k=1}^{\tilde{n}_{i}} a\left(v_{i}\left(x_{i}^{k}\right) \phi_{i}^{k}, v\right) \leq \sum_{k=1}^{\tilde{n}_{i}} a^{1 / 2}\left(v_{i}\left(x_{i}^{k}\right) \phi_{i}^{k}, v_{i}\left(x_{i}^{k}\right) \phi_{i}^{k} v\right) a^{1 / 2}\left(P_{i}^{k} v, P_{i}^{k} v\right) \\
& \leq\left(\sum_{k=1}^{\tilde{n}_{i}} a\left(v_{i}\left(x_{i}^{k}\right) \phi_{i}^{k}, v_{i}\left(x_{i}^{k}\right) \phi_{i}^{k} v\right)\right)^{1 / 2}\left(\sum_{k=1}^{\tilde{n}_{i}} a\left(P_{i}^{k} v, v\right)\right)^{1 / 2} .
\end{aligned}
$$

Combining (4.7) and (4.8) yields

$$
\begin{aligned}
& a(v, v)=\sum_{i=0}^{J} a\left(v_{i}, v\right) \\
& \leq\left(a\left(v_{0}, v_{0}\right)+\sum_{i=1}^{J} \sum_{k=1}^{\tilde{n}_{i}} a\left(v_{i}\left(x_{i}^{k}\right) \phi_{i}^{k}, v_{i}\left(x_{i}^{k}\right) \phi_{i}^{k}\right)\right)^{1 / 2}\left(a\left(P_{0} v, v\right)+\sum_{i=1}^{J} \sum_{k=1}^{\tilde{n}_{i}} a\left(P_{i}^{k} v, v\right)\right)^{1 / 2} .
\end{aligned}
$$

Since $a\left(\phi_{i}^{k}, \phi_{i}^{k}\right) \approx 1$, there holds

$$
a\left(v_{i}\left(x_{i}^{k}\right) \phi_{i}^{k}, v_{i}\left(x_{i}^{k}\right) \phi_{i}^{k}\right) \approx v_{i}^{2}\left(x_{i}^{k}\right) .
$$

The following inequality has been proved in Lemma 3.3 of [30],

$$
\sum_{i=1}^{J} \sum_{k=1}^{\tilde{n}_{i}} v_{i}^{2}\left(x_{i}^{k}\right) \lesssim a(v, v)
$$

For the initial level, we have

$$
a\left(v_{0}, v_{0}\right)=a\left(\Pi_{0} v, \Pi_{0} v\right) \lesssim a(v, v) .
$$

Thus, we obtain

$$
a\left(v_{0}, v_{0}\right)+\sum_{i=1}^{J} \sum_{k=1}^{\tilde{n}_{i}} v_{i}^{2}\left(x_{i}^{k}\right) \lesssim a(v, v) .
$$

Combining the above inequalities, we conclude that there exists a constant $\tilde{K}_{0}$ independent of mesh sizes and mesh levels such that

$$
a(v, v) \leq \tilde{K}_{0}\left(a\left(P_{0} v, v\right)+\sum_{i=1}^{J} \sum_{k=1}^{\tilde{n}_{i}} a\left(P_{i}^{k} v, v\right)\right) \leq \frac{\tilde{K}_{0}}{\gamma} \sum_{i=0}^{J} a\left(T_{i} v, v\right)=\frac{\tilde{K}_{0}}{\gamma} a(T v, v) .
$$

We obtain the desired result by setting $K_{0}=\tilde{K}_{0} / \gamma$. 
4.1.3. Verification of assumption A3. As a prerequisite to verify assumption A3, we need the following key lemma which has been derived in [30].

LEMMA 4.3. For $i=1, \ldots, J$ let $\mathcal{T}_{i}$ be a refinement of $\mathcal{T}_{i-1}$ by the newest vertex bisection algorithm and let $\Omega_{j}^{k}$ be the support of $\phi_{j}^{k}$. Then, for $x_{j}^{k} \in \tilde{\mathcal{N}}_{j}$ we have

$$
\sum_{i=j+1}^{J} \sum_{\substack{x_{i}^{l} \in \tilde{\mathcal{N}}_{i}, x_{i}^{l} \in \mathcal{E}_{j}^{k}}}\left(\frac{h_{i}^{l}}{h_{j}^{k}}\right)^{3 / 2} \lesssim 1 \quad, \quad \sum_{\substack{i=j+1 \\ x_{i}^{l} \in \tilde{\mathcal{N}}_{i}, x_{i}^{l} \in \Omega_{j}^{k}}}^{J}\left(\frac{h_{i}^{l}}{h_{j}^{k}}\right)^{3} \lesssim 1,
$$

where $\mathcal{E}_{j}^{k}=\mathcal{E}_{j}\left(\Omega_{j}^{k}\right)$. Moreover, for $x_{i}^{l} \in \tilde{\mathcal{N}}_{i}$ there holds

$$
\sum_{j=1}^{i-1} \sum_{\substack{x_{j}^{k} \in \tilde{\mathcal{N}}_{j}, x_{i}^{l} \in \mathcal{E}_{j}^{k}}}\left(\frac{h_{i}^{l}}{h_{j}^{k}}\right)^{1 / 2} \lesssim 1 \quad, \quad \sum_{\substack{j=1 \\ x_{j}^{k} \in \tilde{\mathcal{N}}_{j}, x_{i}^{l} \in \Omega_{j}^{k}}}^{i-1}\left(\frac{h_{i}^{l}}{h_{j}^{k}}\right)^{1 / 2} \lesssim 1 .
$$

Now we are in a position to verify assumption A3.

LEMmA 4.4. There exists a constant $K_{1}$ independent of mesh sizes and mesh levels such that assumption A3 holds true.

Proof. In view of (4.4), we have

$$
\begin{aligned}
\sum_{i=1}^{J} \sum_{j=1}^{i-1} a\left(T_{i} v, T_{j} u\right) & =\gamma^{2} \sum_{j=1}^{J} \sum_{i=j+1}^{J} \sum_{k=1}^{\tilde{n}_{j}} a\left(P_{j}^{k} u, \sum_{l=1}^{\tilde{n}_{i}} P_{i}^{l} v\right) \\
& =\gamma^{2} \sum_{j=1}^{J} \sum_{k=1}^{\tilde{n}_{j}} a\left(P_{j}^{k} u, \sum_{i=j+1}^{J} \sum_{l=1}^{\tilde{n}_{i}} P_{i}^{l} v\right) .
\end{aligned}
$$

Setting $\omega=\sum_{i=j+1}^{J} \sum_{l=1}^{\tilde{n}_{i}} P_{i}^{l} v$, there holds

$$
a\left(P_{j}^{k} u, \omega\right)=a\left(P_{j}^{k} u, P_{j}^{k} \omega\right) \leq a^{1 / 2}\left(P_{j}^{k} u, P_{j}^{k} u\right) a^{1 / 2}\left(P_{j}^{k} \omega, P_{j}^{k} \omega\right)
$$

whence

$$
\sum_{i=1}^{J} \sum_{j=1}^{i-1} a\left(T_{i} v, T_{j} u\right) \leq \gamma^{2}\left(\sum_{j=1}^{J} \sum_{k=1}^{\tilde{n}_{j}} a\left(P_{j}^{k} u, P_{j}^{k} u\right)\right)^{1 / 2}\left(\sum_{j=1}^{J} \sum_{k=1}^{\tilde{n}_{j}} a\left(P_{j}^{k} \omega, P_{j}^{k} \omega\right)\right)^{1 / 2} .
$$

It is obvious that

$$
\gamma \sum_{j=1}^{J} \sum_{k=1}^{\tilde{n}_{j}} a\left(P_{j}^{k} u, P_{j}^{k} u\right)=\gamma \sum_{j=1}^{J} \sum_{k=1}^{\tilde{n}_{j}} a\left(P_{j}^{k} u, u\right)=\sum_{j=1}^{J} a\left(T_{j} u, u\right) .
$$

We also have

$$
\gamma \sum_{j=1}^{J} \sum_{k=1}^{\tilde{n}_{j}} a\left(P_{j}^{k} \omega, P_{j}^{k} \omega\right) \lesssim \sum_{i=2}^{J} a\left(T_{i} v, v\right)
$$

We note that

$$
a\left(\phi_{j}^{k}, \phi_{j}^{k}\right) \approx 1 \quad \text { and } \quad P_{j}^{k} P_{i}^{l} v=\frac{a\left(P_{i}^{l} v, \phi_{j}^{k}\right)}{a\left(\phi_{j}^{k}, \phi_{j}^{k}\right)} \phi_{j}^{k} \approx a\left(P_{i}^{l} v, \phi_{j}^{k}\right) \phi_{j}^{k},
$$

and hence,

$$
a\left(P_{j}^{k} \omega, P_{j}^{k} \omega\right) \approx\left(\sum_{i=j+1}^{J} \sum_{l=1}^{\tilde{n}_{i}} a\left(P_{i}^{l} v, \phi_{j}^{k}\right)\right)^{2}
$$


Furthermore, due to

$$
P_{i}^{l} v=\frac{a\left(v, \phi_{i}^{l}\right)}{a\left(\phi_{i}^{l}, \phi_{i}^{l}\right)} \phi_{i}^{l} \approx a\left(v, \phi_{i}^{l}\right) \phi_{i}^{l}
$$

it follows that

$$
a\left(P_{i}^{l} v, \phi_{j}^{k}\right) \approx a\left(a\left(v, \phi_{i}^{l}\right) \phi_{i}^{l}, \phi_{j}^{k}\right)=a\left(\phi_{i}^{l}, \phi_{j}^{k}\right) a\left(v, \phi_{i}^{l}\right)
$$

Since $\phi_{j}^{k}$ is conforming and piecewise linear on $\left.\mathcal{T}_{j}\right|_{\Omega_{j}^{k}}$, we obtain

$$
a\left(\phi_{i}^{l}, \phi_{j}^{k}\right)=\sum_{\substack{T \subset \Omega_{j}^{k}, T \in \mathcal{T}_{j}}} \int_{T} a(x) \nabla \phi_{i}^{l} \cdot \nabla \phi_{j}^{k}=\sum_{\substack{T \subset \Omega_{j}^{k} \\ T \in \mathcal{T}_{j}}} \int_{\partial T} a(x) \frac{\partial \phi_{j}^{k}}{\partial n} \phi_{i}^{l}-\sum_{\substack{T \subset \Omega_{j}^{k} \\ T \in \mathcal{T}_{j}}} \int_{T}\left(\nabla a(x) \cdot \nabla \phi_{j}^{k}\right) \phi_{i}^{l} .
$$

Note that

$$
a\left(v, \phi_{i}^{l}\right)=a\left(P_{i}^{l} v, \phi_{i}^{l}\right) \leq a^{1 / 2}\left(P_{i}^{l} v, P_{i}^{l} v\right) a^{1 / 2}\left(\phi_{i}^{l}, \phi_{i}^{l}\right) \lesssim a^{1 / 2}\left(P_{i}^{l} v, v\right) .
$$

Moreover, observing $\left|\frac{\partial \phi_{j}^{k}}{\partial n}\right| \lesssim\left(h_{j}^{k}\right)^{-1}$ and (2.3), it follows that

$$
\sum_{\substack{x_{i}^{l} \in \tilde{\mathcal{N}}_{i}, x_{i}^{l} \in \Omega_{j}^{k}}} a\left(\phi_{i}^{l}, \phi_{j}^{k}\right) a\left(v, \phi_{i}^{l}\right) \lesssim \sum_{\substack{x_{i}^{l} \in \tilde{\mathcal{N}}_{i}, x_{i}^{l} \in \mathcal{E}_{j}^{k}}} \frac{h_{i}^{l}}{h_{j}^{k}} a^{1 / 2}\left(P_{i}^{l} v, v\right)+\sum_{\substack{x_{i}^{l} \in \tilde{\mathcal{N}}_{i}, x_{i}^{l} \in \Omega_{j}^{k}}} \frac{\left(h_{i}^{l}\right)^{2}}{h_{j}^{k}} a^{1 / 2}\left(P_{i}^{l} v, v\right)
$$

Consequently, in view of (4.11) we get

$$
\begin{aligned}
& a\left(P_{j}^{k} \omega, P_{j}^{k} \omega\right) \lesssim\left(\sum_{i=j+1}^{J} \sum_{\substack{x_{i}^{l} \in \tilde{\mathcal{N}}_{i}, x_{i}^{l} \in \Omega_{j}^{k}}} a\left(\phi_{i}^{l}, \phi_{j}^{k}\right) a\left(v, \phi_{i}^{l}\right)\right)^{2} \\
& \lesssim\left(\sum_{i=j+1}^{J} \sum_{\substack{x_{i}^{l} \in \tilde{\mathcal{N}}_{i}, x_{i}^{l} \in \mathcal{E}_{j}^{k}}} \frac{h_{i}^{l}}{h_{j}^{k}} a^{1 / 2}\left(P_{i}^{l} v, v\right)\right)^{2}+\left(\sum_{\substack{i=j+1 \\
x_{i}^{l} \in \tilde{\mathcal{N}}_{i}, x_{i}^{l} \in \Omega_{j}^{k}}}^{J} \frac{\left(h_{i}^{l}\right)^{2}}{h_{j}^{k}} a^{1 / 2}\left(P_{i}^{l} v, v\right)\right)^{2} \\
& \lesssim\left(\sum_{i=j+1}^{J} \sum_{\substack{x_{i}^{l} \in \tilde{\mathcal{N}}_{i}, x_{i}^{l} \in \mathcal{E}_{j}^{k}}}\left(\frac{h_{i}^{l}}{h_{j}^{k}}\right)^{1 / 2} a\left(P_{i}^{l} v, v\right)\right) \cdot\left(\sum_{\substack { i=j+1 \\
\begin{subarray}{c}{x_{i}^{l} \in \tilde{\mathcal{N}}_{i}, x_{i}^{l} \in \mathcal{E}_{j}^{k}{ i = j + 1 \\
\begin{subarray} { c } { x _ { i } ^ { l } \in \tilde { \mathcal { N } } _ { i } , \\
x _ { i } ^ { l } \in \mathcal { E } _ { j } ^ { k } } }\end{subarray}}^{J}\left(\frac{h_{i}^{l}}{h_{j}^{k}}\right)^{3 / 2}\right) \\
& +\left(\sum_{i=j+1}^{J} \sum_{\substack{x_{i}^{l} \in \tilde{\mathcal{N}}_{i}, x_{i}^{l} \in \Omega_{j}^{k}}} \frac{h_{i}^{l}}{\sqrt{h_{j}^{k}}} a\left(P_{i}^{l} v, v\right)\right) \cdot\left(\sum_{\substack { i=j+1 \\
\begin{subarray}{c}{x_{i}^{l} \in \tilde{\mathcal{N}}_{i}, x_{i}^{l} \in \Omega_{j}^{k}{ i = j + 1 \\
\begin{subarray} { c } { x _ { i } ^ { l } \in \tilde { \mathcal { N } } _ { i } , \\
x _ { i } ^ { l } \in \Omega _ { j } ^ { k } } }\end{subarray}}^{J}\left(\frac{h_{i}^{l}}{\sqrt{h_{j}^{k}}}\right)^{3}\right) \\
& \lesssim\left(\sum_{i=j+1}^{J} \sum_{\substack{x_{i}^{l} \in \tilde{\mathcal{N}}_{i}, x_{i}^{l} \in \mathcal{E}_{j}^{k}}}\left(\frac{h_{i}^{l}}{h_{j}^{k}}\right)^{1 / 2} a\left(P_{i}^{l} v, v\right)+\sum_{\substack{i=j+1 \\
x_{i}^{l} \in \tilde{\mathcal{N}}_{i}, x_{i}^{l} \in \Omega_{j}^{k}}}^{J} \frac{h_{i}^{l}}{\sqrt{h_{j}^{k}}} a\left(P_{i}^{l} v, v\right)\right)\left(1+\left(h_{j}^{k}\right)^{3 / 2}\right) \\
& \lesssim \sum_{i=j+1}^{J} \sum_{\substack{x_{i}^{l} \in \tilde{\mathcal{N}}_{i}, x_{i}^{l} \in \mathcal{E}_{j}^{k}}}\left(\frac{h_{i}^{l}}{h_{j}^{k}}\right)^{1 / 2} a\left(P_{i}^{l} v, v\right)+\sum_{\substack{i=j+1 \\
x_{i}^{l} \in \tilde{\mathcal{N}}_{i}, x_{i}^{l} \in \Omega_{j}^{k}}}^{J} \frac{h_{i}^{l}}{\sqrt{h_{j}^{k}}} a\left(P_{i}^{l} v, v\right) .
\end{aligned}
$$

We set $\delta\left(x_{i}^{l}, x_{j}^{k}\right)=1$, if $x_{i}^{l} \in \mathcal{E}_{j}^{k}$, and $\delta\left(x_{i}^{l}, x_{j}^{k}\right)=0$, otherwise, as well as $\tilde{\delta}\left(x_{i}^{l}, x_{j}^{k}\right)=1$, if $x_{i}^{l} \in \Omega_{j}^{k}$, 
and $\tilde{\delta}\left(x_{i}^{l}, x_{j}^{k}\right)=0$, otherwise. In view of (4.12), we obtain

$$
\begin{aligned}
& \sum_{j=1}^{J} \sum_{k=1}^{\tilde{n}_{j}} a\left(P_{j}^{k} \omega, P_{j}^{k} \omega\right) \lesssim \sum_{j=1}^{J} \sum_{k=1}^{\tilde{n}_{j}} \sum_{i=j+1}^{J} \sum_{\substack{x_{i}^{l} \in \tilde{\mathcal{N}}_{i}, x_{i}^{l} \in \mathcal{E}_{j}^{k}}}\left(\frac{h_{i}^{l}}{h_{j}^{k}}\right)^{1 / 2} a\left(P_{i}^{l} v, v\right)+\sum_{j=1}^{J} \sum_{k=1}^{\tilde{n}_{j}} \sum_{i=j+1}^{J} \sum_{\substack{x_{i}^{l} \in \tilde{\mathcal{N}}_{i}, x_{i}^{l} \in \Omega_{j}^{k}}} \frac{h_{i}^{l}}{\sqrt{h_{j}^{k}}} a\left(P_{i}^{l} v, v\right) \\
& =\sum_{i=2}^{J} \sum_{x_{i}^{l} \in \tilde{\mathcal{N}}_{i}}\left(\sum_{j=1}^{i-1} \sum_{x_{j}^{k} \in \tilde{\mathcal{N}}_{j}}\left(\frac{h_{i}^{l}}{h_{j}^{k}}\right)^{1 / 2} \delta\left(x_{i}^{l}, x_{j}^{k}\right)\right) a\left(P_{i}^{l} v, v\right)+\sum_{i=2}^{J} \sum_{x_{i}^{l} \in \tilde{\mathcal{N}}_{i}}\left(\sum_{j=1}^{i-1} \sum_{x_{j}^{k} \in \tilde{\mathcal{N}}_{j}} \frac{h_{i}^{l}}{\sqrt{h_{j}^{k}}} \tilde{\delta}\left(x_{i}^{l}, x_{j}^{k}\right)\right) a\left(P_{i}^{l} v, v\right) \\
& \lesssim \sum_{i=2}^{J} \sum_{x_{i}^{l} \in \tilde{\mathcal{N}}_{i}} a\left(P_{i}^{l} v, v\right)\left(1+\sqrt{h_{i}^{l}}\right) \lesssim \sum_{i=2}^{J} \sum_{x_{i}^{l} \in \tilde{\mathcal{N}}_{i}} a\left(P_{i}^{l} v, v\right) .
\end{aligned}
$$

This completes the proof of (4.15). Combining (4.13)-(4.15), we deduce

$$
\sum_{i=1}^{J} \sum_{j=1}^{i-1} a\left(T_{i} v, T_{j} u\right) \lesssim\left(\sum_{i=2}^{J} a\left(T_{i} v, v\right)\right)^{1 / 2}\left(\sum_{j=1}^{J} a\left(T_{j} u, u\right)\right)^{1 / 2} .
$$

A similar analysis can be done to derive

$$
\sum_{i=1}^{J} a\left(T_{i} v, T_{0} u\right) \lesssim\left(\sum_{i=1}^{J} a\left(T_{i} v, v\right)\right)^{1 / 2} a\left(T_{0} u, u\right)^{1 / 2} .
$$

Together with (4.16), this inequality provides the assertion. $\square$

4.2. Local Gauss-Seidel smoother. We will now apply the abstract theory to the local Gauss-Seidel smoother $R_{i}$ which is defined by

$$
R_{i}:=\left(I-E_{i}^{\tilde{n}_{i}}\right) A_{i}^{-1},
$$

where $E_{i}^{\tilde{n}_{i}}=\left(I-P_{i}^{\tilde{n}_{i}}\right) \cdots\left(I-P_{i}^{1}\right)=\prod_{k=1}^{\tilde{n}_{i}}\left(I-P_{i}^{k}\right)$. For simplicity, we set $E_{i}:=E_{i}^{\tilde{n}_{i}}$, since no confusion is possible. It is easy to see that

$$
T_{0}=P_{0} \quad, \quad T_{i}=R_{i} A_{i} P_{i}=\left(I-E_{i}\right) P_{i}=I-E_{i} \quad, \quad i=1, \ldots, J .
$$

The decomposition of $v$ is the same as in (4.2). The following identity plays a key role in the subsequent analysis.

Lemma 4.5. For $i=1, \ldots, J$, there holds

$$
a(v, u)-a\left(E_{i} v, E_{i} u\right)=\sum_{k=1}^{\tilde{n}_{i}} a\left(P_{i}^{k} E_{i}^{k-1} v, E_{i}^{k-1} u\right) \quad, \quad v, u \in V_{J},
$$

where $E_{i}^{0}=I$ and $E_{i}^{k-1}$ is defined by

$$
E_{i}^{k-1}:=\left(I-P_{i}^{k-1}\right) \cdots\left(I-P_{i}^{1}\right) \quad, \quad k=2, \ldots, \tilde{n}_{i} .
$$

Proof. Obviously, there holds

$$
E_{i}^{k-1}-E_{i}^{k}=P_{i}^{k} E_{i}^{k-1},
$$

and hence,

$$
I-E_{i}=\sum_{k=1}^{\tilde{n}_{i}} P_{i}^{k} E_{i}^{k-1}
$$


Note that

$$
a\left(E_{i}^{k-1} v, E_{i}^{k-1} u\right)=a\left(E_{i}^{k} v, E_{i}^{k} u\right)+a\left(P_{i}^{k} E_{i}^{k-1} v, P_{i}^{k} E_{i}^{k-1} u\right)
$$

which implies

$$
a(v, u)-a\left(E_{i} v, E_{i} u\right)=\sum_{k=1}^{\tilde{n}_{i}} a\left(P_{i}^{k} E_{i}^{k-1} v, E_{i}^{k-1} u\right) .
$$

Hence, (4.19) is verified.

4.2.1. Verification of assumption A1. We consider the case $i \geq 1$, since assumption A1 is obviously true for $T_{0}$.

Lemma 4.6. Let $T_{i}, i \geq 1$, be defined by (4.18). Then, $T_{i}$ is nonnegative on $V_{J}$ and there holds

$$
a\left(T_{i} v, T_{i} v\right) \leq \omega_{i} a\left(T_{i} v, v\right) \quad, \quad v \in V_{J} \quad, \quad \omega_{i}<2
$$

Proof. Recalling $T_{i}=I-E_{i}, i \geq 1$ and (4.20), we obtain

$$
a\left(T_{i} v, T_{i} v\right)=a\left(\left(I-E_{i}\right) v,\left(I-E_{i}\right) v\right)=\sum_{k, m=1}^{\tilde{n}_{i}} a\left(P_{i}^{k} E_{i}^{k-1} v, P_{i}^{m} E_{i}^{m-1} v\right) .
$$

Using Lemma 4.5 and the same techniques as in (4.6), we deduce

$$
\begin{aligned}
& a\left(T_{i} v, T_{i} v\right) \leq C_{i} \sum_{k=1}^{\tilde{n}_{i}} a\left(P_{i}^{k} E_{i}^{k-1} v, E_{i}^{k-1} v\right) \\
& =C_{i}\left(a(v, v)-a\left(E_{i} v, E_{i} v\right)\right)=C_{i}\left(2 a\left(T_{i} v, v\right)-a\left(T_{i} v, T_{i} v\right)\right),
\end{aligned}
$$

whence

$$
a\left(T_{i} v, T_{i} v\right) \leq \frac{2 C_{i}}{C_{i}+1} a\left(T_{i} v, v\right) .
$$

This implies nonnegativeness of $T_{i}$. Setting $\omega_{i}=\left(2 C_{i}\right) /\left(C_{i}+1\right)<2$ completes the proof. $\square$

\subsubsection{Verification of assumption A2.}

LEMMA 4.7. Let $\left\{T_{i}, i=0,1, \ldots, J\right\}$ be given as in (4.18). Then, there exists a constant $K_{0}$ such that

$$
a(v, v) \leq K_{0} a(T v, v) \quad, \quad v \in V_{J} .
$$

Proof. From the decomposition of $v$ in $(4.2)$, it follows that $a(v, v)=\sum_{i=0}^{J} a\left(v_{i}, v\right)$. Similar to (4.8), for $i=1, \ldots, J$ we also have

$$
a\left(v_{i}, v\right) \leq\left(\sum_{k=1}^{\tilde{n}_{i}} a\left(v_{i}\left(x_{i}^{k}\right) \phi_{i}^{k}, v_{i}\left(x_{i}^{k}\right) \phi_{i}^{k}\right)\right)^{1 / 2}\left(\sum_{k=1}^{\tilde{n}_{i}} a\left(P_{i}^{k} v, P_{i}^{k} v\right)\right)^{1 / 2}
$$

Due to the identity $I-E_{i}^{k-1}=\sum_{m=1}^{k-1} P_{i}^{m} E_{i}^{m-1}$, we deduce

$$
\begin{aligned}
& \sum_{k=1}^{\tilde{n}_{i}} a\left(P_{i}^{k} v, P_{i}^{k} v\right)=\sum_{k=1}^{\tilde{n}_{i}} a\left(P_{i}^{k} v, P_{i}^{k} E_{i}^{k-1} v\right)+\sum_{k=1}^{\tilde{n}_{i}} \sum_{m=1}^{k-1} a\left(P_{i}^{k} v, P_{i}^{k} P_{i}^{m} E_{i}^{m-1} v\right) \\
& \leq\left(\sum_{k=1}^{\tilde{n}_{i}} a\left(P_{i}^{k} v, P_{i}^{k} v\right)\right)^{1 / 2}\left(\sum_{k=1}^{\tilde{n}_{i}} a\left(P_{i}^{k} E_{i}^{k-1} v, E_{i}^{k-1} v\right)\right)^{1 / 2}+\sum_{k, m=1}^{\tilde{n}_{i}}\left|a\left(P_{i}^{k} v, P_{i}^{m} E_{i}^{m-1} v\right)\right| .
\end{aligned}
$$


Furthermore, by Hölder's inequality and (4.6)

$$
\sum_{k, m=1}^{\tilde{n}_{i}}\left|a\left(P_{i}^{k} v, P_{i}^{m} E_{i}^{m-1} v\right)\right| \lesssim\left(\sum_{k=1}^{\tilde{n}_{i}} a\left(P_{i}^{k} v, P_{i}^{k} v\right)\right)^{1 / 2}\left(\sum_{k=1}^{\tilde{n}_{i}} a\left(P_{i}^{k} E_{i}^{k-1} v, E_{i}^{k-1} v\right)\right)^{1 / 2}
$$

Then, it follows from (4.21) that

$$
\sum_{k=1}^{\tilde{n}_{i}} a\left(P_{i}^{k} v, P_{i}^{k} v\right) \lesssim \sum_{k=1}^{\tilde{n}_{i}} a\left(P_{i}^{k} E_{i}^{k-1} v, E_{i}^{k-1} v\right) \lesssim a\left(T_{i} v, v\right),
$$

whence

$$
a\left(P_{0} v, P_{0} v\right)+\sum_{k=1}^{\tilde{n}_{i}} a\left(P_{i}^{k} v, P_{i}^{k} v\right) \lesssim \sum_{i=0}^{J} a\left(T_{i} v, v\right) .
$$

Similar to the analysis of (4.9) and (4.10), we deduce that assumption A2 holds true.

\subsubsection{Verification of assumption A3.}

LEMMA 4.8. There exists a constant $K_{1}$ independent of mesh sizes and mesh levels such that assumption $\mathbf{A} 3$ holds true for $\left\{T_{i}, i=0,1, \ldots, J\right\}$ defined by (4.18).

Proof. For $\xi_{i}=T_{i} v$, it follows from (4.18) that

$$
\begin{aligned}
& \sum_{i=1}^{J} \sum_{j=1}^{i-1} a\left(T_{i} v, T_{j} u\right)=\sum_{j=1}^{J} \sum_{i=j+1}^{J} a\left(\xi_{i},\left(I-E_{j}\right) u\right) \\
& =\sum_{j=1}^{J} \sum_{i=j+1}^{J} \sum_{k=1}^{\tilde{n}_{j}} a\left(P_{j}^{k} \xi_{i}, P_{j}^{k} E_{j}^{k-1} u\right)=\sum_{j=1}^{J} \sum_{k=1}^{\tilde{n}_{j}} a\left(P_{j}^{k} \sum_{i=j+1}^{J} \xi_{i}, P_{j}^{k} E_{j}^{k-1} u\right) .
\end{aligned}
$$

By Hölder's inequality there holds

$$
\begin{aligned}
& \sum_{i=1}^{J} \sum_{j=1}^{i-1} a\left(T_{i} v, T_{j} u\right) \\
& \leq\left(\sum_{j=1}^{J} \sum_{k=1}^{\tilde{n}_{j}} a\left(P_{j}^{k} E_{j}^{k-1} u, E_{j}^{k-1} u\right)\right)^{1 / 2}\left(\sum_{j=1}^{J} \sum_{k=1}^{\tilde{n}_{j}} a\left(\sum_{i=j+1}^{J} P_{j}^{k} \xi_{i}, \sum_{i=j+1}^{J} P_{j}^{k} \xi_{i}\right)\right)^{1 / 2} .
\end{aligned}
$$

Next, we show that

$$
\sum_{j=1}^{J} \sum_{k=1}^{\tilde{n}_{j}} a\left(\sum_{i=j+1}^{J} P_{j}^{k} \xi_{i}, \sum_{i=j+1}^{J} P_{j}^{k} \xi_{i}\right) \lesssim \sum_{i=2}^{J} a\left(T_{i} v, v\right) .
$$

Due to

$$
P_{j}^{k} \xi_{i}=\frac{a\left(\xi_{i}, \phi_{j}^{k}\right)}{a\left(\phi_{j}^{k}, \phi_{j}^{k}\right)} \phi_{j}^{k} \approx a\left(\xi_{i}, \phi_{j}^{k}\right) \phi_{j}^{k} \quad, \quad P_{i}^{l} E_{i}^{l-1} v=\frac{a\left(E_{i}^{l-1} v, \phi_{i}^{l}\right)}{a\left(\phi_{i}^{l}, \phi_{i}^{l}\right)} \phi_{i}^{l} \approx a\left(E_{i}^{l-1} v, \phi_{i}^{l}\right) \phi_{i}^{l},
$$

we have

$$
a\left(\sum_{i=j+1}^{J} P_{j}^{k} \xi_{i}, \sum_{i=j+1}^{J} P_{j}^{k} \xi_{i}\right) \approx\left(\sum_{i=j+1}^{J} a\left(\xi_{i}, \phi_{j}^{k}\right)\right)^{2},
$$

and

$$
a\left(\xi_{i}, \phi_{j}^{k}\right)=a\left(\left(I-E_{i}\right) v, \phi_{j}^{k}\right)=\sum_{l=1}^{\tilde{n}_{i}} a\left(P_{i}^{l} E_{i}^{l-1} v, \phi_{j}^{k}\right) \approx \sum_{l=1}^{\tilde{n}_{i}} a\left(\phi_{i}^{l}, \phi_{j}^{k}\right) a\left(E_{i}^{l-1} v, \phi_{i}^{l}\right) .
$$


By Lemma 4.3 and a similar technique as in the previous subsection, we obtain

$$
\begin{aligned}
& \sum_{j=1}^{J} \sum_{k=1}^{\tilde{n}_{j}}\left(\sum_{i=j+1}^{J} a\left(\xi_{i}, \phi_{j}^{k}\right)\right)^{2} \\
\lesssim & \sum_{j=1}^{J} \sum_{k=1}^{\tilde{n}_{j}} \sum_{i=j+1}^{J} \sum_{x_{i}^{l} \in \tilde{\mathcal{N}}_{i},}\left(\frac{h_{i}^{l}}{h_{j}^{k}}\right)^{1 / 2} a\left(P_{i}^{l} E_{i}^{l-1} v, E_{i}^{l-1} v\right) \\
& +\sum_{j=1}^{J} \sum_{k=1}^{\tilde{n}_{j}} \sum_{i=j+1}^{J} \sum_{x_{i}^{l} \in \tilde{\mathcal{N}}_{i},} \frac{h_{i}^{l}}{\sqrt{h_{j}^{k}}} a\left(P_{i}^{l} E_{i}^{l-1} v, E_{i}^{l-1} v\right) \\
\lesssim & \sum_{i=2}^{J} \sum_{x_{i}^{l} \in \tilde{N}_{i}^{k}} a\left(P_{i}^{l} E_{i}^{l-1} v, E_{i}^{l-1} v\right) \sum_{j=1}^{i-1} \sum_{x_{j}^{k} \in \tilde{N}_{j}}\left(\frac{h_{i}^{l}}{h_{j}^{k}}\right)^{1 / 2} \delta\left(x_{i}^{l}, x_{j}^{k}\right) \\
& +\sum_{i=2}^{J} \sum_{x_{i}^{l} \in \tilde{N}_{i}} a\left(P_{i}^{l} E_{i}^{l-1} v, E_{i}^{l-1} v\right) \sum_{j=1}^{i-1} \sum_{x_{j}^{k} \in \tilde{N}_{j}} \frac{h_{i}^{l}}{\sqrt{h_{j}^{k}}} \tilde{\delta}\left(x_{i}^{l}, x_{j}^{k}\right) \\
\lesssim & \sum_{i=2}^{J} \sum_{x_{i}^{l} \in \tilde{N}_{i}} a\left(P_{i}^{l} E_{i}^{l-1} v, E_{i}^{l-1} v\right)\left(1+\sqrt{h_{i}^{l}}\right) \lesssim \sum_{i=2}^{J} a\left(T_{i} v, v\right) .
\end{aligned}
$$

Hence, (4.24) is verified. In view of (4.19), (4.21), (4.23) and (4.24), it follows that

$$
\sum_{i=1}^{J} \sum_{j=1}^{i-1} a\left(T_{i} v, T_{j} u\right) \lesssim\left(\sum_{i=2}^{J} a\left(T_{i} v, v\right)\right)^{1 / 2}\left(\sum_{j=1}^{J} a\left(T_{j} u, u\right)\right)^{1 / 2} .
$$

Moreover, we deduce

$$
\sum_{i=1}^{J} a\left(T_{i} v, T_{0} u\right) \lesssim\left(\sum_{i=1}^{J} a\left(T_{i} v, v\right)\right)^{1 / 2} a\left(T_{0} u, u\right)^{1 / 2}
$$

which, together with (4.25), allows to conclude.

Next, we show that (4.1) holds true for the Gauss-Seidel case. Actually, similar to the Jacobi case, by (4.22) we have

$$
\begin{aligned}
a(T v, u) & =a\left(P_{0} v, P_{0} u\right)+\sum_{i=1}^{J} \sum_{k=1}^{\tilde{n}_{i}} a\left(P_{i}^{k} E_{i}^{k-1} v, P_{i}^{k} u\right) \\
& \leq a\left(P_{0} v, v\right)^{1 / 2} a\left(P_{0} u, u\right)^{1 / 2}+\sum_{i=1}^{J} \sum_{k=1}^{\tilde{n}_{i}} a\left(P_{i}^{k} E_{i}^{k-1} v, E_{i}^{k-1} v\right)^{1 / 2} a\left(P_{i}^{k} u, P_{i}^{k} u\right)^{1 / 2} \\
& \leq\left(a\left(P_{0} v, v\right)+\sum_{i=1}^{J} \sum_{k=1}^{\tilde{n}_{i}} a\left(P_{i}^{k} E_{i}^{k-1} v, E_{i}^{k-1} v\right)\right)^{1 / 2}\left(a\left(P_{0} u, u\right)+\sum_{i=1}^{J} \sum_{k=1}^{\tilde{n}_{i}} a\left(P_{i}^{k} u, P_{i}^{k} u\right)\right)^{1 / 2} \\
& \lesssim\left(\sum_{i=0}^{J} a\left(T_{i} v, v\right)\right)^{1 / 2}\left(\sum_{i=0}^{J} a\left(T_{i} u, u\right)\right)^{1 / 2} .
\end{aligned}
$$

5. Hierarchical basis multilevel method. In this section, we will discuss the hierarchical basis multigrid method (HBMG) and the hierarchical basis preconditioning method (HBP) on locally refined meshes. The hierarchical basis method is based on the decomposition of $V_{J}$ into 
subspaces given by

$$
V_{J}=\sum_{i=0}^{J} \bar{V}_{i}, \bar{V}_{0}=I_{0} V_{J} \quad, \quad \bar{V}_{i}=\left(I_{i}-I_{i-1}\right) V_{J} \quad, \quad i=1, \ldots, J .
$$

Here, $I_{i}: V_{J} \rightarrow V_{i}$ is the nodal value interpolation.

By means of the above decomposition, we can derive the convergence result for HBMG by verifying assumptions A1-A3 as in section 4. Compared with the above local multigrid method, the smoothing operator $R_{i}(1 \leq i \leq J)$ in HBMG is carried out only on the set of new nodes, e.g., $\overline{\mathcal{N}}_{i}=\mathcal{N}_{i} \backslash \mathcal{N}_{i-1}$. We set $\bar{n}_{i}=\# \overline{\mathcal{N}}_{i}$. The operators $A_{i}, P_{i}, Q_{i}$ are all well defined by the subspaces $\left\{\bar{V}_{i}: i=0,1, \ldots, J\right\}$.

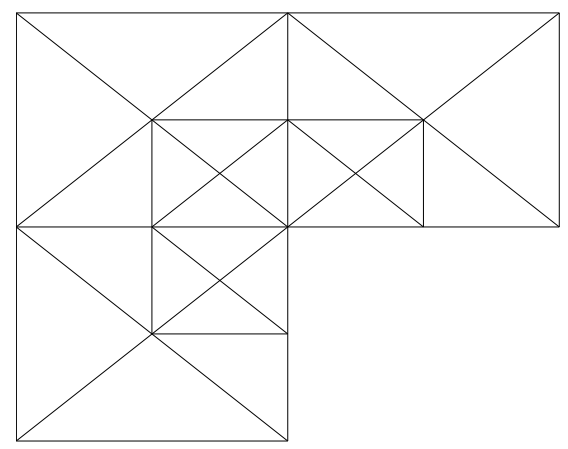

The coarse level $\mathcal{T}_{i-1}$

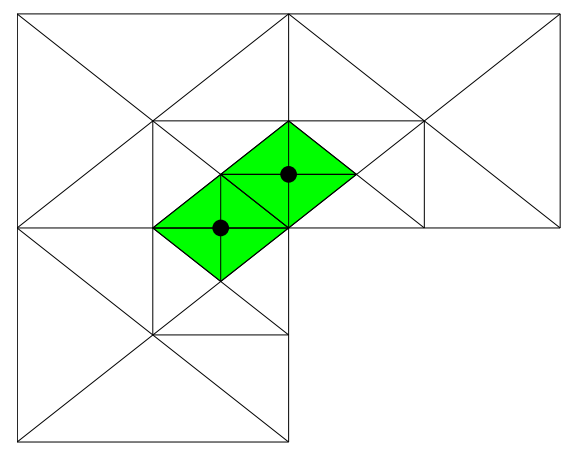

The fine level $\mathcal{T}_{i}$

FIG 5.1. Illustration of $\overline{\mathcal{N}}_{i}$ : the big dots in the right figure refer to $\overline{\mathcal{N}}_{i}$.

For brevity, we only provide the convergence analysis of HBMG for local Jacobi smoothing. A similar analysis can be carried out for HBMG in case of local Gauss-Seidel smoothing. We note that the assumptions $\mathbf{A} \mathbf{1}$ and $\mathbf{A} \mathbf{3}$ can be verified as in Lemma 4.1 and Lemma 4.4. For the stability assumption A2, we have the following result.

Lemma 5.1. There exists a constant $K_{0}$ such that

$$
a(v, v) \leq K_{0}\left(1+\left|\log h_{\min }\right|\right)^{2} a(T v, v) \quad, \quad v \in V_{J},
$$

where $h_{\min }=\min \left\{h_{T}, T \in \mathcal{T}_{J}\right\}$.

Proof. In view of the decomposition (5.1), it follows that

$$
v_{i}=\left(I_{i}-I_{i-1}\right) v=\sum_{k=1}^{\bar{n}_{i}} v\left(x_{i}^{k}\right) \phi_{i}^{k} \quad, \quad i=1, \ldots, J .
$$

Similar to (4.9), we deduce

$$
\begin{aligned}
& a(v, v)=\sum_{i=0}^{J} a\left(v_{i}, v\right) \\
& \leq\left(a\left(v_{0}, v_{0}\right)+\sum_{i=1}^{J} \sum_{k=1}^{\bar{n}_{i}} a\left(v\left(x_{i}^{k}\right) \phi_{i}^{k}, v\left(x_{i}^{k}\right) \phi_{i}^{k}\right)\right)^{1 / 2}\left(a\left(P_{0} v, v\right)+\sum_{i=1}^{J} \sum_{k=1}^{\bar{n}_{i}} a\left(P_{i}^{k} v, v\right)\right)^{1 / 2} .
\end{aligned}
$$

Since $a\left(v_{0}, v_{0}\right)=a\left(I_{0} v, I_{0} v\right) \lesssim a(v, v),(5.2)$ is proved, if we can show that

$$
\sum_{i=1}^{J} \sum_{k=1}^{\bar{n}_{i}} a\left(v\left(x_{i}^{k}\right) \phi_{i}^{k}, v\left(x_{i}^{k}\right) \phi_{i}^{k}\right) \lesssim\left(1+\left|\log h_{\min }\right|\right)^{2} a(v, v) .
$$


For any triangle $K \subset \Omega_{i}^{k} \cap \mathcal{T}_{i}$, let $C$ be a constant representing the $L^{2}$ projection of $v$ onto $K$. We recall the following inequality [34]

$$
\|v-C\|_{0, \infty, K} \lesssim\left(\log \frac{h_{i}^{k}}{h_{\min }}+1\right)^{1 / 2}|v|_{1, K} .
$$

Then, we have

$$
\begin{aligned}
& \left|\left(I_{i}-I_{i-1}\right) v\right|_{1, K}=\left|\left(I_{i}-I_{i-1}\right)(v-C)\right|_{1, K}=\left|\left(I-I_{i-1}\right) I_{i}(v-C)\right|_{1, K} \\
& \lesssim\left|I_{i}(v-C)\right|_{1, K} \lesssim\|v-C\|_{0, \infty, K} \lesssim\left(\log \frac{h_{i}^{k}}{h_{\min }}+1\right)^{1 / 2}|v|_{1, K} .
\end{aligned}
$$

Hence, it follows from (5.5) that

$$
\sum_{i=1}^{J} \sum_{k=1}^{\bar{n}_{i}} a\left(v\left(x_{i}^{k}\right) \phi_{i}^{k}, v\left(x_{i}^{k}\right) \phi_{i}^{k}\right) \lesssim \sum_{i=1}^{J} \sum_{k=1}^{\bar{n}_{i}}\left|\left(I_{i}-I_{i-1}\right) v\right|_{1, \Omega_{i}^{k}}^{2} \lesssim \sum_{i=1}^{J} \sum_{k=1}^{\bar{n}_{i}}\left(\log \frac{h_{i}^{k}}{h_{\min }}+1\right)|v|_{1, \Omega_{i}^{k}}^{2} .
$$

For the sequence $\left\{\hat{\mathcal{T}}_{i}, i=0,1,2, \ldots\right\}$, obtained by uniform bisection from the initial mesh $\hat{\mathcal{T}}_{0}=\mathcal{T}_{0}$, we denote by $\hat{\mathcal{N}}_{i}$ the set of interior nodes of $\hat{\mathcal{T}}_{i}$, and we set $\hat{h}_{i}=\left(\frac{1}{2}\right)^{i} h_{0}$. It has been shown in [30] that

$$
h_{i}(z) \approx \hat{h}_{\rho_{i}(z)} \quad, \quad z \in \hat{\mathcal{N}}_{\rho_{i}(z)},
$$

where

$$
\rho_{i}(z)=\left[\frac{\log \left(h_{i}(z) / h_{0}\right)}{\log (1 / 2)}\right]
$$

We define

$$
\sigma(m, z)=\left\{i: z \in \overline{\mathcal{N}}_{i}, \rho_{i}(z)=m, 0 \leq i \leq J\right\} .
$$

It has been shown in [30] that $\# \sigma(m, z) \lesssim 1$. Let $B\left(z, c \hat{h}_{m}\right)=\left\{x \in \Omega:|x-z|<c \hat{h}_{m}\right\}$ and $M:=\max _{z \in \mathcal{N}_{J}} \rho_{J}(z)$. Then, $h_{\min } \approx \hat{h}_{M}$. Combining (5.6) and (5.7), we have

$$
\begin{aligned}
& \sum_{i=1}^{J} \sum_{k=1}^{\bar{n}_{i}} a\left(v\left(x_{i}^{k}\right) \phi_{i}^{k}, v\left(x_{i}^{k}\right) \phi_{i}^{k}\right) \lesssim \sum_{i=1}^{J} \sum_{k=1}^{\bar{n}_{i}}\left(\log \frac{\hat{h}_{\rho_{i}\left(x_{i}^{k}\right)}}{h_{\min }}+1\right)|v|_{1, \Omega_{i}^{k}}^{2} \\
& \lesssim \sum_{m=0}^{M} \sum_{\substack{z \in \tilde{\mathcal{N}}_{i}, \rho_{i}(z)=m \\
1 \leq i \leq J}}\left(\log \frac{\hat{h}_{m}}{h_{\min }}+1\right)|v|_{1, B\left(z, c \hat{h}_{m}\right)}^{2}=\sum_{m=0}^{M}\left(\log \frac{\hat{h}_{m}}{h_{\min }}+1\right) \sum_{\substack{z \in \hat{\mathcal{N}}_{m} \cap \mathcal{N}_{J} \\
i \in \sigma(m, z)}}|v|_{1, B\left(z, c \hat{h}_{m}\right)}^{2} \\
& \lesssim \sum_{m=0}^{M}\left(\log \hat{h}_{m}-\log h_{\min }+1\right)|v|_{1, \Omega}^{2} \lesssim\left(1+\left|\log h_{\min }\right|\right)^{2}|v|_{1, \Omega}^{2},
\end{aligned}
$$

which completes the proof of the lemma.

Finally, we have the following convergence result for the algorithm HBMG.

ThEOREM 5.1. For the algorithm HBMG with local Jacobi or local Gauss-Seidel smoothing, the norm of the error operator $E$ can be bounded as follows

$$
a(E v, E v) \leq \delta a(v, v) \quad, \quad v \in V_{J},
$$

where

$$
\delta=1-\frac{2-\omega}{K_{0}\left(1+\left|\log h_{\min }\right|\right)^{2}\left(1+K_{1}\right)^{2}} \quad, \quad \omega=\max _{i}\left\{\omega_{i}\right\} .
$$


Hence,

$$
\left\|I-B_{J} A_{J}\right\|_{a}=\left\|E E^{*}\right\|_{a} \leq \delta .
$$

Next, we provide a condition number estimate for the hierarchical basis preconditioning method. Let

$$
\|\| v \|_{*}^{2}=\sum_{z \in \mathcal{N}_{0}}\left(I_{0} v\right)^{2}(z)+\sum_{i=1}^{J} \sum_{z \in \mathcal{N}_{i} \backslash \mathcal{N}_{i-1}}\left|\left(I_{i}-I_{i-1}\right) v(z)\right|^{2} .
$$

Similar to (5.4), the following upper bound holds true

$$
\left.|||v|\right|_{*} ^{2} \lesssim\left(1+\left|\log h_{\min }\right|\right)^{2}|v|_{1, \Omega}^{2} .
$$

A lower bound can be derived as in the verification of assumption A3:

$$
|v|_{1, \Omega}^{2} \lesssim \||| v||_{*}^{2} .
$$

THEOREM 5.2. Let $\hat{A}_{J}$ be the stiffness matrix based on the hierarchical basis on locally refined meshes $\mathcal{T}_{J}$. Then, there holds

$$
\operatorname{cond}\left(\hat{A}_{J}\right) \leq C\left(1+\left|\log h_{\min }\right|\right)^{2},
$$

where the constant $C$ is independent of mesh sizes and mesh levels.

Let $S$ be the matrix which transforms the representations of the finite element functions of $V_{J}$ with respect to the hierarchical basis into the representations with respect to the usual nodal basis. We then have the representation $\hat{A}_{J}=S^{T} A_{J} S$. Since

$$
\operatorname{cond}\left(S^{T} A_{J} S\right)=\operatorname{cond}\left(S S^{T} A_{J}\right),
$$

the hierarchical basis method can be interpreted as a preconditioning method for $A_{J}$ with the preconditioner $S S^{T}$.

6. Numerical results. In this section, we present several examples to illustrate the optimality of algorithm 3.1 and algorithm 3.2. For algorithm 3.2, we test the PCG method for LMAA with local Jabobi smoothing. Furthermore, in order to compare the two methods, we present examples for HBMG and HBP on locally refined meshes. We remark that LMG and HBMG are implemented with $O(N)$ operations each iteration, where $N$ is the number of degrees of freedom (DOFs, i.e., interior nodes or free nodes). As has been pointed out in [6] and [34], the overall complexity of HBMG (the symmetric case, e.g., with local Jacobi smoothing) and the hierarchical basis method used as a preconditioner for CG is $O\left(N \log \left(h_{\min }\right)|\log \epsilon|\right)$ operations, required to reduce the initial error by a given factor $\epsilon$. On the other hand, for LMAA with local Jacobi smoothing as a preconditioner for $\mathrm{CG}, O(N|\log \epsilon|)$ operations are required. The following implementation is based on the FFW toolbox from [15].

The local error estimator for each element is defined as in [22]. The stopping rule for algorithm 3.1 is as follows: At the i-th level, let $u_{i}^{0}=u_{i-1}, r_{i}^{n}=f_{i}-A_{i} u_{i}^{n}$. Then, the multigrid iteration stops when the following relation is satisfied

$$
\frac{\left\|r_{i}^{n}\right\|_{0, \Omega}}{\left\|r_{i}^{0}\right\|_{0, \Omega}} \leq 10^{-8}
$$

For the PCG method, the stopping criterion is as follows

$$
\left\|r_{i}^{0}-A_{i} r_{i}^{n}\right\|_{0, \Omega} \leq \text { tol }\left\|r_{i}^{0}\right\|_{0, \Omega} \quad, \quad \text { tol }=10^{-8},
$$

where $\left\{r_{i}^{k}: k=1,2, \ldots\right\}$ denotes the set of iterative solutions of the residual equation $A_{i} x=r_{i}^{0}$. 
EXAMPLE 6.1. Consider the following elliptic boundary value problem with Dirichlet boundary conditions on the L-shaped domain $\Omega=[-1,1] \times[-1,1] \backslash(0,1] \times[-1,0)$.

$$
\begin{aligned}
-\Delta u+0.5 u & =f(x, y) \quad \text { in } \Omega, \\
u & =g(x, y) \quad \text { on } \partial \Omega,
\end{aligned}
$$

where $f$ and $g$ are chosen such that the exact solution in polar coordinates is given by $u(r, \theta)=$ $r^{\frac{2}{3}} \sin \left(\frac{2}{3} \theta\right)$.

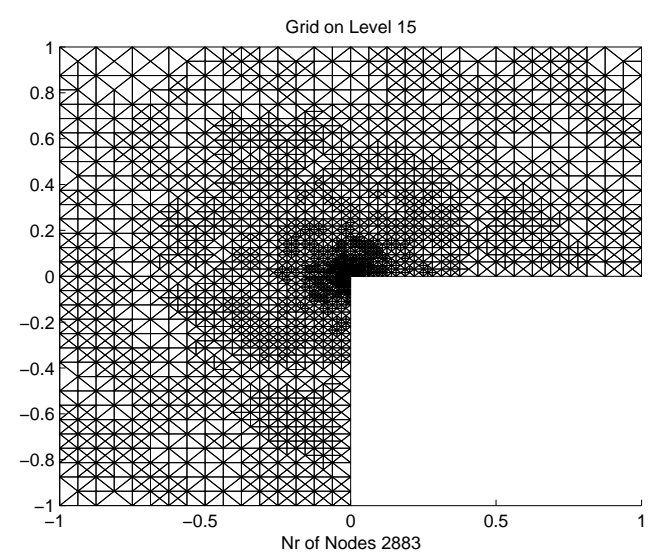

FIG 6.1. The locally refined mesh after 15 refinement steps (Example 6.1)

\begin{tabular}{|c|c|c|c|c|}
\hline Level & DOFs & LMG-Jacobi & LMG-GS & HBMG-GS \\
\hline 15 & 2718 & 25 & 13 & 51 \\
\hline 17 & 5255 & 23 & 11 & 59 \\
\hline 21 & 18242 & 22 & 11 & 75 \\
\hline 25 & 58758 & 23 & 12 & 88 \\
\hline 27 & 102397 & 22 & 11 & 88 \\
\hline 29 & 173698 & 20 & 10 & 90 \\
\hline 31 & 290479 & 20 & 10 & 101 \\
\hline 33 & 487869 & 19 & 10 & 113 \\
\hline 35 & 799086 & 17 & 9 & 118 \\
\hline
\end{tabular}

Table 6.1. Iteration steps on each level for the algorithms under comparison

We first present the numerical results for algorithm 3.1 and HBMG. We refer to LMGJacobi as algorithm 3.1 with local Jacobi smoothing $(\gamma=0.8)$, to LMG-GS as algorithm 3.1 with local Gauss-Seidel smoothing and to HBMG-GS as the hierarchical basis multigrid method with local Gauss-Seidel smoothing. Table 6.1, Figure 6.2, and Figure 6.3 show that the number of iterations and the convergence rate, i.e., the reduction factor $\left\|I-B_{J} A_{J}\right\|_{a}$, of algorithm 3.1 with local Jacobi or local Gauss-Seidel smoothing, per level are all bounded independently of mesh sizes and mesh levels, which confirms our theoretical results. For HBMG-GS, we observe that the number of iterations depends on the mesh levels. Figures 6.2-6.4 also show that the CPU time (in seconds) of each iteration of LMG and HBMG is linear with respect to the DOFs.

Next, we study the performance of algorithm 3.2 and the hierarchical basis preconditioning method (HBP). As can be seen from Table 6.2, the number of iterations by CG without preconditioning increases fast with the mesh levels. However, for PCG by LMAA with local Jacobi smoothing (LMAA-Jacobi), the iteration steps per level are both independent of mesh sizes and mesh levels. Similar to HBMG-GS, for PCG by HBP, the iteration steps depend on the mesh levels. Figures 6.5 and 6.6 show that for these two algorithms the CPU time (in seconds) of each iteration also is in accordance with the theoretical analysis. 

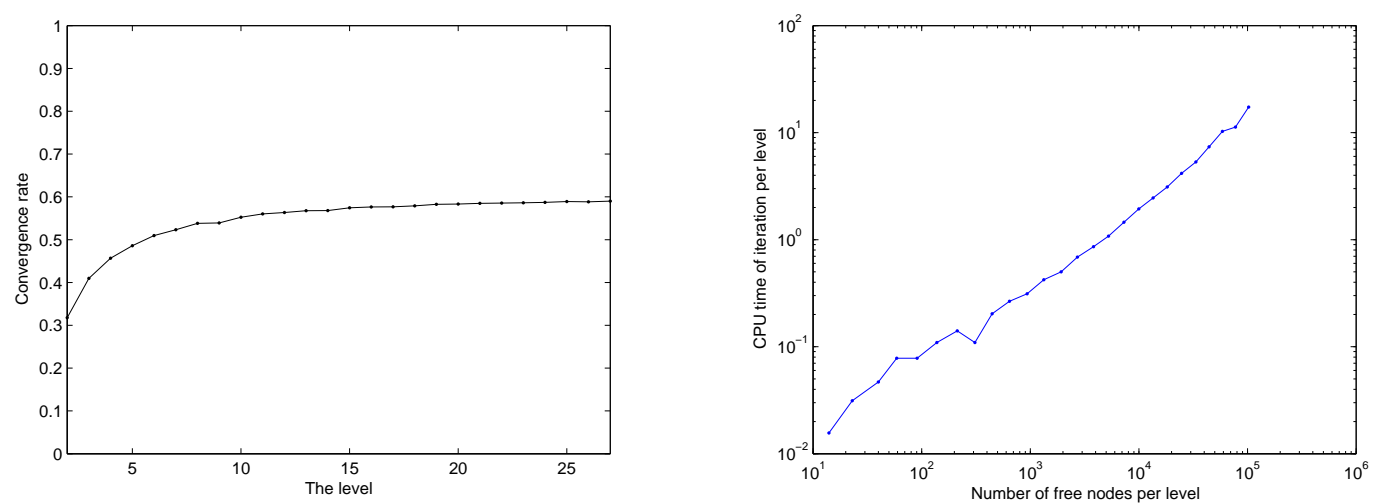

FIG 6.2. Reduction factor (left) and CPU time (right) per level for LMG-Jacobi.
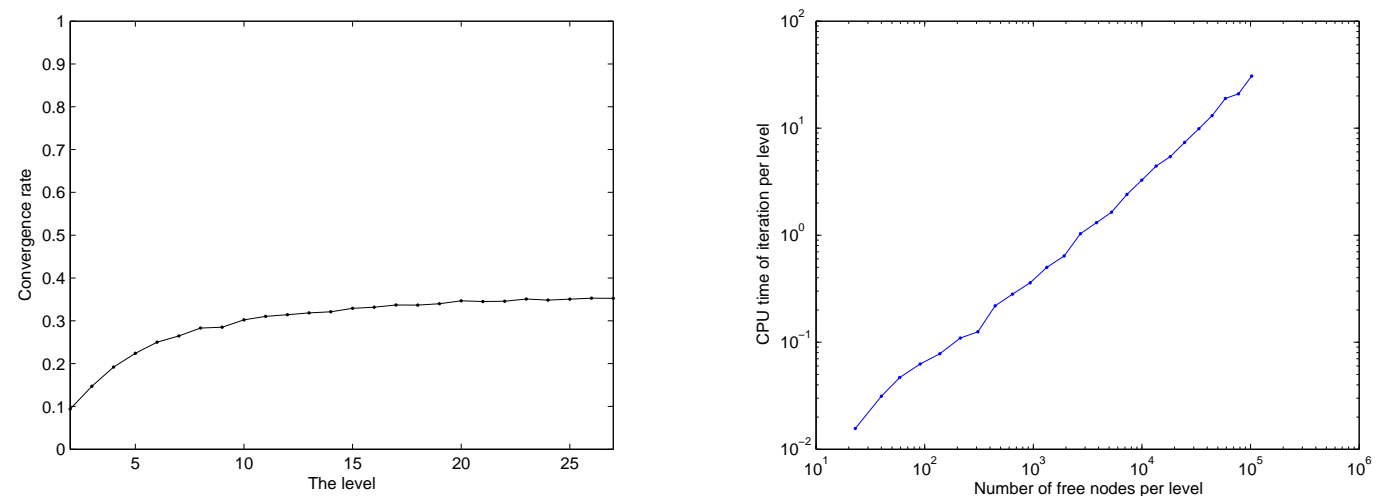

FIG 6.3. Reduction factor (left) and CPU time (right) per level for LMG-GS.
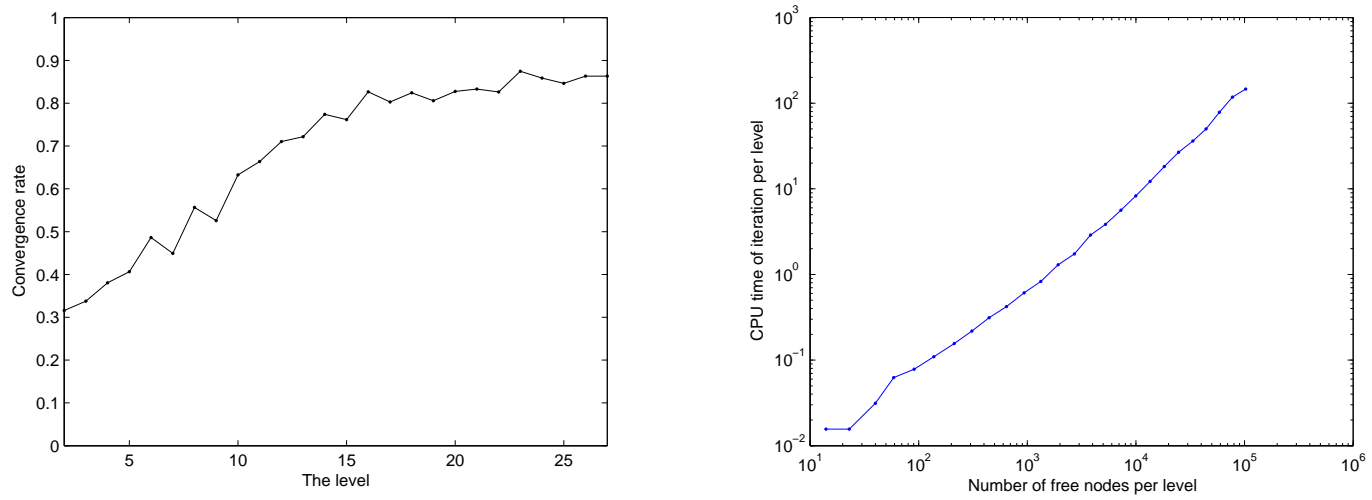

FIG 6.4. Reduction factor $\left\|I-B_{J} A_{J}\right\|_{a}$ (left) and $C P U$ time (right) per level for HBMG with local Gauss-Seidel smoothing. Here, $B_{J}$ and $A_{J}$ are derived based on the hierarchical basis.

EXAMPLE 6.2. Consider Poisson's equation

$$
-\Delta u=1 \quad \text { in } \Omega
$$

with Dirichlet boundary conditions on the slit domain $\Omega=\{(x, y):|x|+|y| \leq 1\} \backslash\{(x, y): 0 \leq x \leq$ $1, y=0\}$. The exact solution (in polar coordinates) is $r^{1 / 2} \sin (\theta / 2)-\frac{1}{4} r^{2}$.

Figure 6.7 displays the locally refined mesh with 1635 nodes after 15 refinement steps. Table 6.3 and Figures 6.8, 6.9 show that the linear increase in CPU time and the convergence rate, i.e., 


\begin{tabular}{|r|c|l|c|c|}
\hline Level & DOFs & CG & LMAA-Jacobi & HBP \\
\hline 16 & 3819 & 185 & 38 & 61 \\
\hline 18 & 7285 & 257 & 39 & 66 \\
\hline 20 & 13524 & 337 & 40 & 71 \\
\hline 22 & 24765 & 446 & 41 & 78 \\
\hline 24 & 44284 & 576 & 42 & 79 \\
\hline 26 & 77431 & 691 & 40 & 85 \\
\hline 28 & 134344 & 965 & 41 & 90 \\
\hline 30 & 224771 & 1220 & 41 & 94 \\
\hline 32 & 376628 & 1415 & 41 & 95 \\
\hline 34 & 625557 & 1739 & 41 & 98 \\
\hline
\end{tabular}

Table 6.2. Iteration steps on each level for the algorithms under comparison

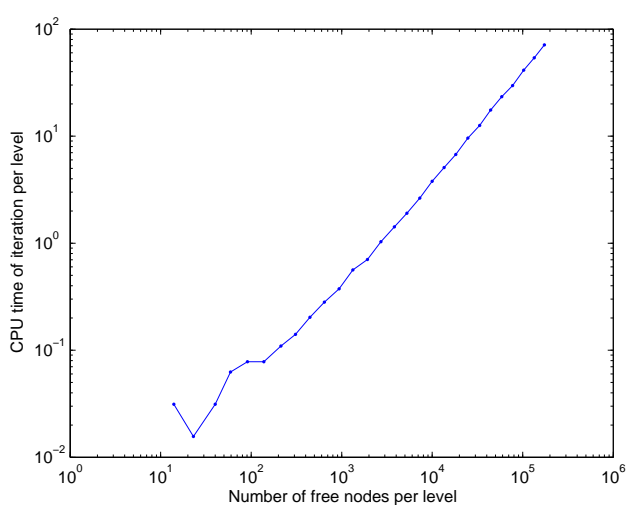

FIG 6.5. CPU time for LMAA-Jacobi

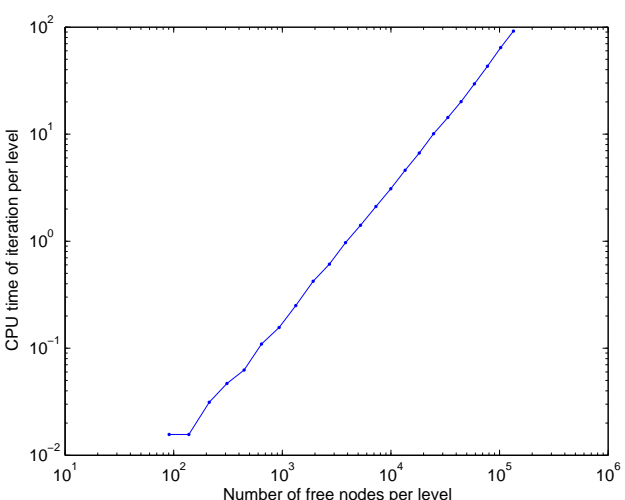

FIG 6.6. CPU time for $H B P$

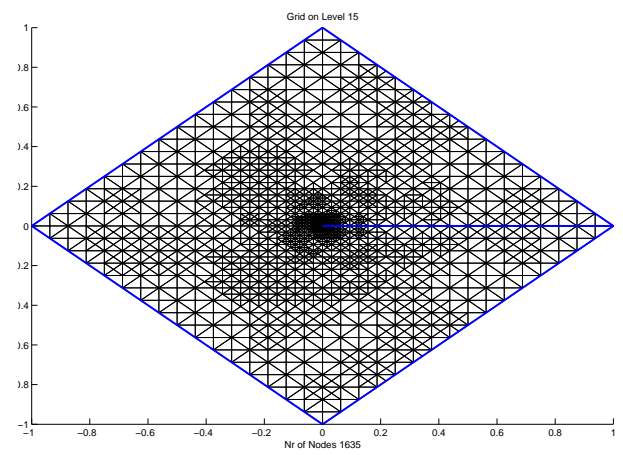

FIG 6.7. The locally refined mesh after 15 refinement steps (Example 6.2)

the reduction factor $\left\|I-B_{J} A_{J}\right\|_{a}$, are bounded independently of mesh sizes and mesh levels, which indicates the optimality of algorithm LMG-Jacobi $(\gamma=0.8)$ and LMG-GS. 


\begin{tabular}{|r|c|c|c|c|}
\hline Level & DOFs & LMG-Jacobi & LMG-GS & HBMG-GS \\
\hline 19 & 6115 & 40 & 20 & 57 \\
\hline 21 & 11713 & 39 & 20 & 63 \\
\hline 25 & 41460 & 38 & 19 & 76 \\
\hline 27 & 74727 & 36 & 18 & 84 \\
\hline 29 & 144648 & 36 & 17 & 93 \\
\hline 31 & 250576 & 35 & 17 & 99 \\
\hline 34 & 619187 & 34 & 17 & 107 \\
\hline 35 & 795755 & 34 & 17 & 107 \\
\hline 36 & 1075195 & 32 & 16 & 115 \\
\hline
\end{tabular}

Table 6.3. Iteration steps on each level for the algorithms under comparison
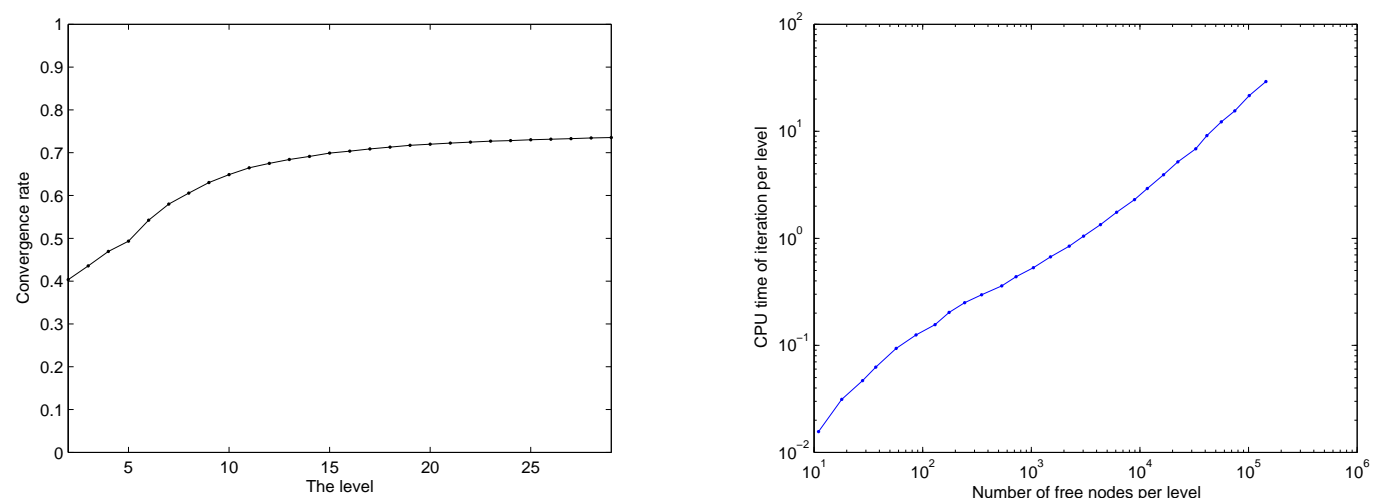

FIG 6.8. Reduction factor and CPU time per level for LMG-Jacobi.
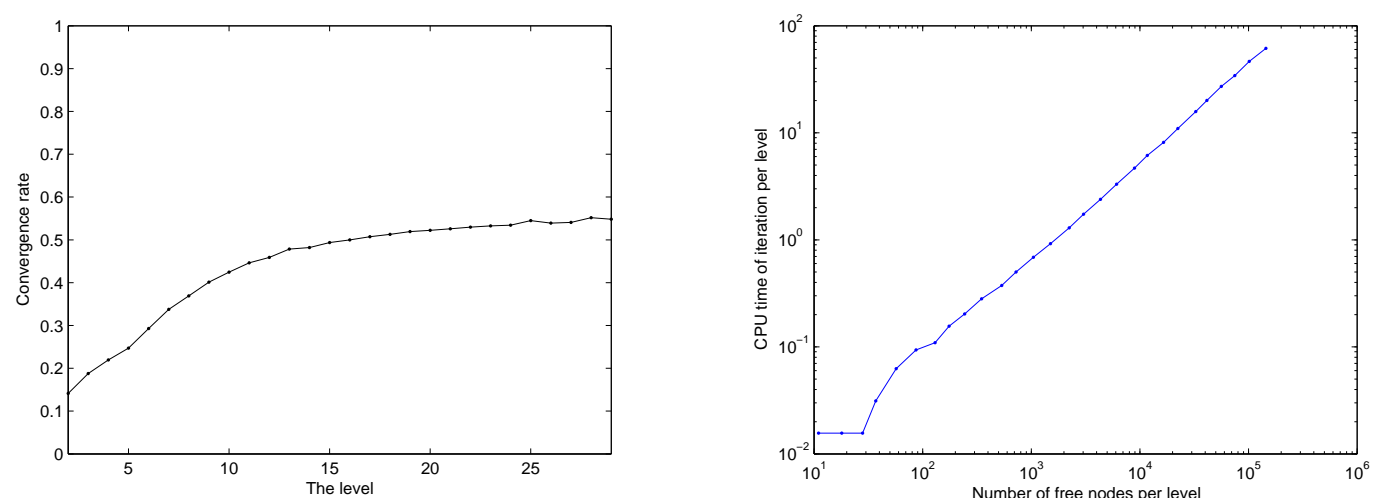

FIG 6.9. Reduction factor and CPU time per level for LMG-GS.

For algorithm 3.2, Table 6.4 and Figure 6.11 show the optimality of algorithm LMAA-Jacobi $(\gamma=0.8)$, whereas Table 6.3, Figure 6.10 and Table 6.4, Figure 6.12 show that the convergence of HBMG-GS and PCG by HBP also depends on the mesh levels. For these two algorithms, the CPU time (in seconds) of each iteration is almost linear with respect to the DOFs. 

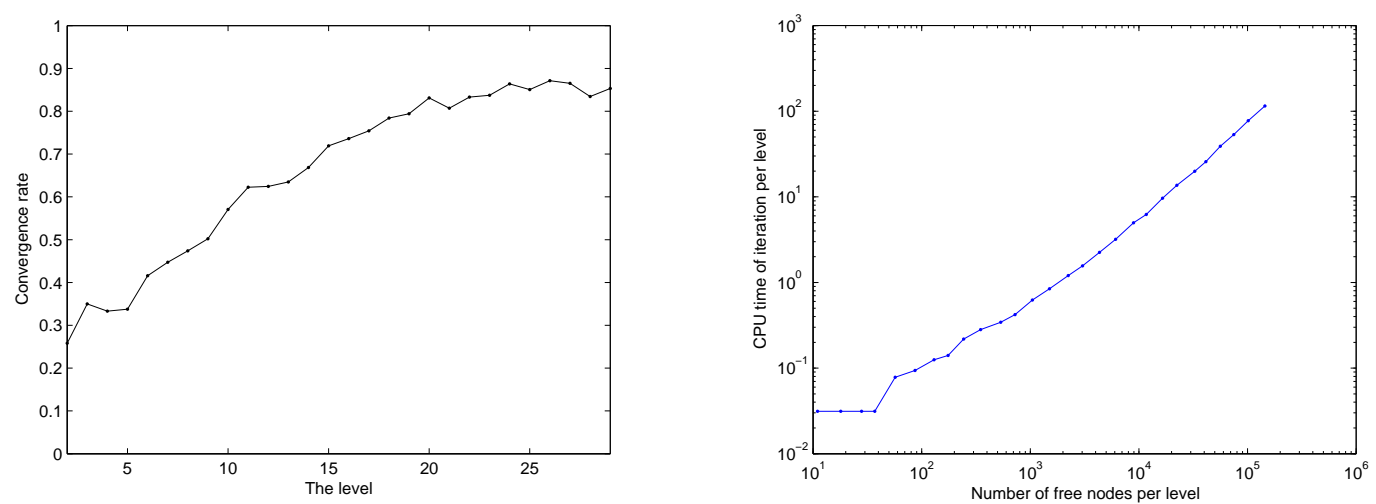

FIG 6.10. Reduction factor and CPU time per level for HBMG-GS.

\begin{tabular}{|r|c|l|c|c|}
\hline Level & DOFs & CG & LMAA-Jacobi & HBP \\
\hline 16 & 2240 & 117 & 39 & 55 \\
\hline 18 & 4338 & 195 & 43 & 61 \\
\hline 20 & 8936 & 269 & 45 & 67 \\
\hline 22 & 16500 & 325 & 47 & 72 \\
\hline 24 & 32733 & 483 & 49 & 76 \\
\hline 26 & 56161 & 618 & 50 & 82 \\
\hline 28 & 101565 & 827 & 51 & 85 \\
\hline 30 & 184049 & 1068 & 50 & 90 \\
\hline 32 & 327094 & 1333 & 52 & 95 \\
\hline 34 & 619187 & 1689 & 53 & 98 \\
\hline
\end{tabular}

Table 6.4. Iteration steps on each level for the algorithms under comparison

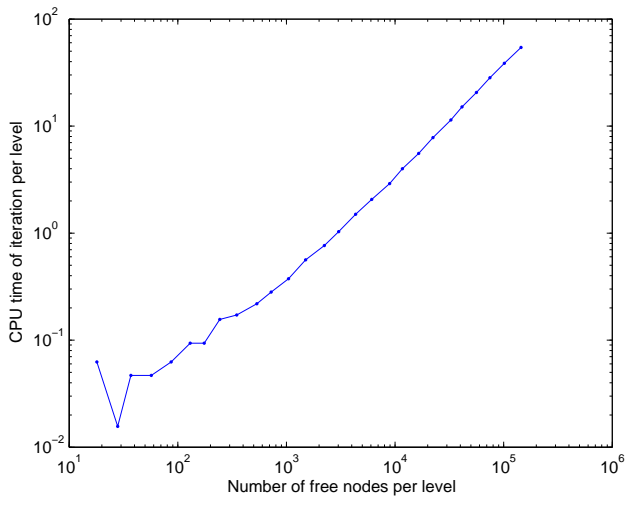

FIG 6.11. CPU time for LMAA-Jacobi

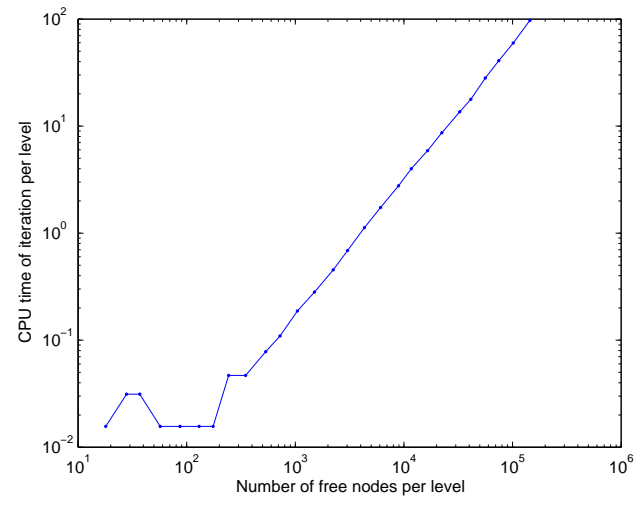

FIG 6.12. $C P U$ time for $H B P$

\section{REFERENCES}

[1] M. Ainsworth and W. Mclean, Multilevel diagonal scaling preconditioners for boundary element equations on locally refined meshes, Numer. Math., 93(2003), 387-413.

[2] B. Aksoylu and M. Holst, An odyssey into local refinement and multilevel preconditioning I: Optimality of the BPX preconditioned, Institute for Computational Engineering and Sciences, The University of Texas at Austin, ICES Technical Report 05-03, 2005.

[3] D. Bai and A. Brandt, Local mesh refinement multilevel techniques, SIAM J. Sci. Stat. Comput., 8(1987), 109-134.

[4] E. Bänsch, Local mesh refinement in 2 and 3 dimensions, Impact of Computing in Science and Engineering, 3(1991), 181-191. 
[5] R.E. Bank and L. R. Scott, On the conditioning of finite element equations with highly refined meshes, SIAM J. Numer. Anal., 26(1989), 1383-1394.

[6] R.E. Bank, T.F. Dupont, and H. Yserentant, The hierarchical basis multigrid method, Numer. Math., 52(1988), 427-458.

[7] P. Binev, W. Dahmen, and R. DeVore, Adaptive finite element methods with convergence rates, Numer. Math., 97(2004), 219-268.

[8] D. Braess and W. Hackbusch, A new convergence proof for the multigrid method including the V-cycle, SIAM J. Numer. Anal., 36(1983), 967-975.

[9] J.H. Bramble, Multigrid Methods, Pitman, Boston, 1993.

[10] J.H. Bramble and J.E. Pasicak, New estimates for multigrid algorithms including the V-cycle, Math. Comp., 60(1993), 447-471.

[11] J.H. Bramble, J.E. Pasicak, J. Wang, and J. Xu, Convergence estimate for multigrid algorithms without regularity assumptions, Math. Comp., 57(1991), 23-45.

[12] J.H. Bramble and J.E. Pasicak, New estimates for multigrid algorithms including the V-cycle, Math. Comp., 60(1993), 447-471.

[13] J.H. Bramble, J.E. Pasicak, J.Wang, and J. Xu, Convergence estimates for product iterative methods with applications to domain decomposition, Math. Comp., 57(1991), 23-45.

[14] S.C. Brenner, Convergence of the multigrid V-cycle algorithms for the second order boundary value problems without full elliptic regularity, Math. Comp., 71(2002), 507-525.

[15] A. Byfut, J. Gedicke, D. Günther, J. Reininghaus, S. Wiedemann, etc, FFW Documentation, Humboldt University of Berlin, Germany, 2007.

[16] J.M. Cascon, C. Kreuzer, R.H. Nochetto, and K.G. Siebert, Quasi-optimal convergence rate for an adaptive finite element method, SIAM J. Numer. Anal., 46(2008), 2524-2550.

[17] P.G. Ciarlet, The Finite Element Method for Elliptic Problems, North-Holland, Amsterdam, 1978.

[18] W. Dahmen and A. Kunoth, Multilevel preconditioning, Numer. Math., 63(1992), 315-344.

[19] W. Hackbusch, Multigrid Methods and Applications. Springer, Berlin-Heidelberg-New York, 1985.

[20] S. McCormick, Fast adaptive composite grid (FAC) methods, in: Defect Correction Methods: Theory and Applications, K. Böhmer and H.J. Stetter, eds., Computing Supplementum 5, Wien: Sringer Verlag, 1984, 115-121.

[21] S. McCormick and J. Thomas, The fast adaptive composite grid (FAC) method for elliptic equations, Math. Comp., 46(1986), 439-456.

[22] P. Morin, R.H. Nochetto, and K.G. Siebert, Convergence of adaptive finite element methods, SIAM Review, 44(2002), 631-658.

[23] W.F. Mitchell, Optimal multilevel iterative methods for adaptive grids, SIAM J.Sci.Stat.Comput., 13(1992), 146-167.

[24] P. Oswald, Multilevel Finite Element Approximation: Theory and Applications. Teubner, Stuttgart, 1994.

[25] P. Oswald, Multilevel solvers for elliptic problems on domains, in: MultiscaleWavelet Methods for PDEs, W. Dahmen, A.J. Kurdila and P. Oswald, eds., Academic Press, 1996, 3-58.

[26] U. Rüde, Fully adaptive multigrid methods, SIAM J. Numer. Anal., 30(1993), 230-248.

[27] R. Scott and S. Zhang, Finite element interpolation of nonsmooth functions satisfying boundary conditions, Math. Comp., 54(1990), 483-493.

[28] R. Stevenson, Optimality of a standard adaptive finite element method, Foundations of Computional Mathematics, 2(2007), 245-269.

[29] A. Toselli and O. Widlund, Domain Decomposition Methods - Algorithms and Theory, Springer, Berlin-Heidelberg-New York, 2005.

[30] H.J. Wu and Z.M. Chen, Uniform convergence of multigrid V-cycle on adaptively refined finite element meshes for second order elliptic problems, Science in China, 39(2006), 1405-1429.

[31] O.B. Widlund, Optimal iterative refinement methods, in: Domain Decomposition Methods, T. Chan, R. Glowinski, J. Périaux and O. Widlund, eds., SIAM, Philadelphia, 1989, 114-125.

[32] J. Xu, Iterative methods by space decomposition and subspace correction, SIAM Review, 34(1992), 581-613.

[33] J. Xu and L. Zikatanov, The method of alternating projections and the method of subspace corrections in Hilbert space, J.Amer.Math.Soc., 15(2002), 573-597.

[34] H. Yserentant, On the multi-level splitting of finite element spaces, Numer. Math., 49(1986), 379-412.

[35] H. Yserentant, Two preconditioners based on the multi-level splitting of finite element spaces, Numer. Math., 58(1990), 163-184.

[36] H. Yserentant, Old and new convergence proofs for multigrid methods, Acta Numerica, 2(1993), 285-326. 